\title{
WestVirginiaUniversity
}

THE RESEARCH REPOSITORY @ WVU

Graduate Theses, Dissertations, and Problem Reports

2014

\section{From Graph Coloring to Receptor Clustering}

Ye Chen

West Virginia University

Follow this and additional works at: https://researchrepository.wvu.edu/etd

\section{Recommended Citation}

Chen, Ye, "From Graph Coloring to Receptor Clustering" (2014). Graduate Theses, Dissertations, and Problem Reports. 385.

https://researchrepository.wvu.edu/etd/385

This Dissertation is protected by copyright and/or related rights. It has been brought to you by the The Research Repository @ WVU with permission from the rights-holder(s). You are free to use this Dissertation in any way that is permitted by the copyright and related rights legislation that applies to your use. For other uses you must obtain permission from the rights-holder(s) directly, unless additional rights are indicated by a Creative Commons license in the record and/ or on the work itself. This Dissertation has been accepted for inclusion in WVU Graduate Theses, Dissertations, and Problem Reports collection by an authorized administrator of The Research Repository @ WVU.

For more information, please contact researchrepository@mail.wvu.edu. 


\title{
From Graph Coloring to Receptor Clustering
}

\author{
Ye Chen \\ Dissertation submitted to the \\ Eberly College of Arts and Sciences \\ at West Virginia University \\ in partial fulfillment of the requirements \\ for the degree of \\ Doctor of Philosophy \\ in \\ Mathematics \\ Adam Halasz, Ph.D., co-Chair \\ Hong-Jian Lai, Ph.D., co-Chair \\ John Goldwasser, Ph.D. \\ Guodong Guo, Ph.D. \\ Jerzy Wojciechowski, Ph.D. \\ Cun-Quan Zhang, Ph.D. \\ Department of Mathematics \\ West Virginia University \\ Morgantown, West Virginia 26506 \\ 2014
}




\section{ABSTRACT \\ From Graph Coloring to Receptor Clustering \\ Ye Chen}

This dissertation focuses on two topics, hued coloring in graph theory and receptor clustering analysis in computational biology.

\section{Hued colorings for planar graphs, graphs of higher genus and $K_{4}$-minor free graphs}

For integers $k, r>0$, a $(k, r)$-coloring of a graph $G$ is a proper coloring of the vertices of $G$ with $k$ colors such that every vertex $v$ of degree $d(v)$ is adjacent to vertices with at least $\min \{d(v), r\}$ different colors. The r-hued chromatic number, denoted by $\chi_{r}(G)$, is the smallest integer $k$ for which a graph $G$ has a $(k, r)$-coloring. A list assignment $L$ of $G$ is a function that assigns to every vertex $v$ of $G$ a set $L(v)$ of positive integers. For a given list assignment $L$ of $G$, an $(L, r)$-coloring of $G$ is a proper coloring $c$ of the vertices such that every vertex $v$ of degree $d(v)$ is adjacent to vertices with at least $\min \{d(v), r\}$ different colors and $c(v) \in L(v)$. The $r$-hued choice number of $G, \chi_{L, r}(G)$, is the least integer $k$ such that every list assignment $L$ with $|L(v)|=k, \forall v \in V(G)$, permits an $(L, r)$-coloring. It is known that for any graph $G, \chi_{r}(G) \leq \chi_{L, r}(G)$. Using Euler distributions, we proved the following results, where (ii) and (iii) are best possible.

(i) If $G$ is planar, then $\chi_{L, 2}(G) \leq 6$. Moreover, $\chi_{L, 2}(G) \leq 5$ when $\Delta(G) \leq 4$.

(ii) If $G$ is planar, then $\chi_{2}(G) \leq 5$.

(iii) If $G$ is a graph with genus $g(G) \geq 1$, then $\chi_{L, 2}(G) \leq \frac{1}{2}(7+\sqrt{1+48 g(G)})$. Let $K(r)=r+3$ if $2 \leq r \leq 3$, and $K(r)=\lfloor 3 r / 2\rfloor+1$ if $r \geq 4$. We proved that if $G$ is a $K_{4}$-minor free graph, then

(i) $\chi_{r}(G) \leq K(r)$, and the bound can be attained;

(ii) $\chi_{L, r}(G) \leq K(r)+1$. This extends a previous result in [Discrete Math. 269 (2003) 303-309].

\section{Quantitative description and impact of VEGF receptor clustering}

Cell membrane-bound receptors control signal initiation in many important cellular signaling pathways. Microscopic imaging and modern labeling techniques reveal that certain receptor types tend to co-localize in clusters, ranging from a few to hundreds of members. Here, we further develop a method of defining receptor clusters in the membrane based on their mutual distance, and apply it to a set of transmission microscopy (TEM) images of vascular endothelial growth factor (VEGF) receptors. We clarify the difference between the observed distributions and random placement. Moreover, we outline a model of clustering based on the hypothesis of pre-existing domains that have a high affinity for receptors. The observed results are consistent 
with the combination of two distributions, one corresponding to the placement of clusters, and the other to that of random placement of individual receptors within the clusters. Further, we use the preexisting domain model to calculate the probability distribution of cluster sizes. By comparing to the experimental result, we estimate the likely area and attractiveness of the clustering domains.

Furthermore, as VEGF signaling is involved in the process of blood vessel development and maintenance, it is of our interest to investigate the impact of VEGF receptors (VEGFR) clustering. VEGF signaling is initiated by binding of the bivalent VEGF ligand to the membrane-bound receptors (VEGFR), which in turn stimulates receptor dimerization. To address these questions, we have formulated the simplest possible model. We have postulated the existence of a single high affinity region in the cell membrane, which acts as a transient trap for receptors. We have defined an ODE model by introducing high- and low-density receptor variables and introduce the corresponding reactions from a realistic model of VEGF signal initiation. Finally, we use the model to investigate the relation between the degree of VEGFR concentration, ligand availability, and signaling. In conclusion, our simulation results provide a deeper understanding of the role of receptor clustering in cell signaling. 


\section{Acknowledgements}

First and foremost, I am most indebted to my co-advisors, Dr. Hong-Jian Lai and Dr. Adam Halasz, for their continued encouragement and support over these last few years. It is a pleasure to work under their supervision. Without them, this dissertation could not have come about.

I would like to take this opportunity to thank Dr. Suohai Fan at Jinan University (Guangzhou) who brought me to the research field of combinatorics and graph theory.

I would also like to thank my other committee members: Dr. John Goldwasser, Dr. Guodong Guo, Dr. Jerzy Wojciechowski, and Dr. Cun-Quan Zhang, for their help during my studies.

And finally, I would like to thank the Department of Mathematics and Eberly College of Arts and Sciences at West Virginia University for providing me with an excellent study environment and financial support during my study as a graduate student. 


\section{DEDICATION}

To

my mother Caidi Li , my father Zanqiang Chen,

and

my husband Xiangming $W u$, my sweet son Terrance $W u$ 


\section{Contents}

1 Introduction $\quad 1$

1.1 Notations and terminologies on $r$-hued coloring . . . . . . . . . . . . 1

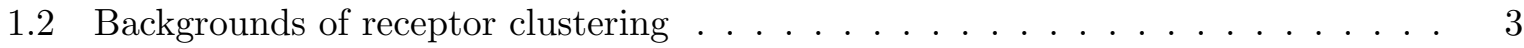

2 2-hued Coloring for Planar Graphs and Graphs of Higher Genus 6

2.1 Priliminaries . . . . . . . . . . . . . . . . . . 6

2.2 2-hued coloring for planar graphs . . . . . . . . . . . . . . 8

2.32 -hued coloring for graphs of higher genus . . . . . . . . . . . . . 11

$3 r$-hued Coloring of $K_{4}$-minor Free Graphs $\quad 16$

3.1 Proof of the main result $\ldots \ldots \ldots \ldots \ldots \ldots$

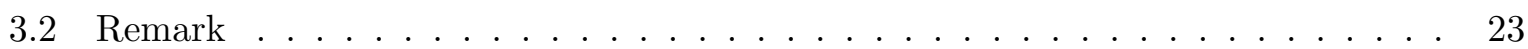

4 The quantitative description of VEGF receptor localization based on hierarchical clustering $\quad 24$

4.1 Methods . . . . . . . . . . . . . . . . . . . . . 24

4.2 Results and discussion . . . . . . . . . . . . . . . . . . . . . 29

5 Quantitative description and impact of VEGF receptor localization 35

5.1 From microscopic details to global behavior . . . . . . . . . . . . . . . . 35

5.2 ODE model and steady states . . . . . . . . . . . . . . . . . 36

5.3 Results and discussion . . . . . . . . . . . . . . . . . . . . 44 


\section{Chapter 1}

\section{Introduction}

\section{$1.1 \quad$ Notations and terminologies on $r$-hued coloring}

Graphs in this paper are simple and finite. For undefined terminologies and notations see [7] and [47]. Thus for a graph $G, \Delta(G), \delta(G)$ and $\chi(G)$ denote the maximum degree, minimum degree and chromatic number of $G$ respectively. For $v \in V(G)$, let $N_{G}(v)$ denote the set of vertices adjacent to $v$ in $G$, and $d_{G}(v)=\left|N_{G}(v)\right|$. Vertices in $N_{G}(v)$ are neighbor of $v$. For an integer $g \geq 0$, let $S_{g}$ be the orientable surface obtained from the sphere by adding $g$ handles, and let $N_{g}$ be the non-orientable surface obtained from the sphere by adding $g$ Möbius strips (cross-caps). Given an embedding of $G$ on a closed surface, the genus $g(G)$ of a graph $G$ is the minimum number $g$ such that $G$ can be embedded on the surface $S_{g}$ or $N_{g}$.

Let $G$ be a graph, $k>0$ be an integer, $\bar{k}=\{1,2, \cdots, k\}$, and $c: V(G) \mapsto \bar{k}$ be a map. For $S \subseteq V(G)$, define $c(S)=\{c(u) \mid u \in S\}$. For integers $k>0$ and $r>0$, a $(k, r)$-coloring of a graph $G$ is a map $c: V(G) \mapsto \bar{k}$ satisfying both the following.

(C1) $c(u) \neq c(v)$, for every edge $u v \in E(G)$;

$(\mathrm{C} 2)\left|c\left(N_{G}(v)\right)\right| \geq \min \left\{d_{G}(v), r\right\}$, for every $v \in V(G)$.

For a fixed integer $r>0$, the $r$-hued chromatic number of $G$, denoted by $\chi_{r}(G)$, is the smallest $k$ such that $G$ has a $(k, r)$-coloring. The concept was first introduced in [30] and [26], where $\chi_{2}(G)$ is called the 2-hued chromatic number of $G$. Later in [25], a referee suggested the name of conditional chromatic number of $G$. Recently, we received several comments on the name of conditional coloring, suggesting that does not reveal the nature of the coloring. Therefore, we decided to use the name $r$-hued chromatic number to reflect the use of many colors near a vertex.

By the definition of $\chi_{r}(G)$, it follows immediately that $\chi(G)=\chi_{1}(G)$, and so $r$-hued coloring is a generalization of the classical graph coloring. Let $G^{2}$ be the graph defined as the following, $V\left(G^{2}\right)=V(G), E\left(G^{2}\right)=\left\{u v \mid d_{G}(u, v) \leq 2\right\}$, then $\chi_{\Delta(G)}(G)=\chi\left(G^{2}\right)$. For any integers $i>j>$ 
0 , any $(k, i)$-coloring of $G$ is also a $(k, j)$-coloring of $G$, and so

$$
\chi(G) \leq \chi_{2}(G) \leq \cdots \leq \chi_{r-1}(G) \leq \chi_{r}(G) \leq \cdots \leq \chi_{\Delta(G)}(G)=\chi_{\Delta(G)+1}(G)=\cdots
$$

A list assignment $L$ of $G$ is a function that assigns to every vertex $v$ of $G$ a set $L(v)$ of positive integers. An $L$-coloring is a proper coloring $c$ such that $c(v) \in L(v)$, for every $v \in V(G)$. Such coloring is also called list coloring. $G$ is said to be $k$-choosable if, for every list assignment $L$ with $|L(v)|=k$, for all $v \in V(G)$, there exists an $L$-coloring of $G$. The list chromatic number $\operatorname{ch}(G)$ of $G$, is the least integer $k$ such that $G$ is $k$-choosable.

There is also a similar generalization for the list coloring. For a given list assignment $L$ of $G$ and a given positive integer $r$, an $r$-hued $L$-coloring $c$ of $G$ is an $L$-coloring of $G$ such that $\left|c\left(N_{G}(v)\right)\right| \geq \min \left\{d_{G}(v), r\right\}$, for every vertex $v \in V(G)$. We call such coloring an $(L, r)$-coloring. The $r$-hued list chromatic number of $G, c h_{r}(G)$, is the least integer $k$ such that $G$ admits an $(L, r)$-coloring, for any list assignment $L$ with $|L(v)|=k$, for every vertex $v \in V(G)$. Similarly, $\operatorname{ch}(G)=\operatorname{ch}_{1}(G)$ and $c h_{\Delta(G)}(G)=\operatorname{ch}\left(G^{2}\right)$. As for any integers $i>j>0$, any $(L, i)$-coloring of $G$ is also an $(L, j)$-coloring of $G$, it follows

$$
\operatorname{ch}(G) \leq \chi L, 2(G) \leq \cdots \leq \operatorname{ch}_{r-1}(G) \leq \operatorname{ch}_{r}(G) \leq \cdots \leq \operatorname{ch}_{\Delta(G)}(G)=\operatorname{ch}_{\Delta(G)+1}(G)=\cdots
$$

For any positive integers $k$ and $r$, let $L(v)=\bar{k}$, for every vertex $v$ of a graph $G$. Then every $(k, r)$-coloring of $G$ is also an $(L, r)$-coloring of $G$, and so

$$
\chi_{r}(G) \leq \operatorname{ch}_{r}(G) .
$$

Some recent results are published for the case $r=2$. In [26], an analogue of Brooks Theorem for $\chi_{2}$ is proved. Akbari et al. [1] proved that $\chi L, 2(G) \leq \Delta(G)+1$ if $G$ has no component isomorphic to $C_{5}$ and if $\Delta(G) \geq 3$. Later in [12], Esperet disproved a conjecture $\chi L, 2(G)=$ $\max \left\{\operatorname{ch}(G), \chi_{2}(G)\right\}$ made in [1]. In [2], Alishahi obtained that $\chi_{2}(G) \leq \chi(G)+14.06 \ln k+1$, for any $k$-regular graph.

The research for general $r$ is also of interest. In [25], it is shown that for $r \geq 2, \chi_{r}(G) \leq$ $\Delta(G)+r^{2}-r+1$ if $\Delta(G) \leq r$. A Moore graph is a regular graph with diameter $d$ and girth $2 d+1$. Ding et al. [10] proved that $\chi_{r}(G) \leq(\Delta(G))^{2}+1$, where equality holds if and only if $G$ is a Moore graph. This is also improved in [28] as $\chi_{r}(G) \leq r(\Delta(G))+1$.

The $r$-hued coloring for graphs $G$ embedded on surfaces is of particular interest. The famous Four Color Theorem [4, 5, 39] and the Heawood formula [20] provide complete answers to the case when $r=1$. Heawood [20] proved that if $G$ is a connected graph with a 2-cell embedding on $S_{g(G)}$, then $\chi(G) \leq \frac{1}{2}(7+\sqrt{1+48 g(G)})$. The main results of 2-hued coloring for planar Graphs and graphs of higher genus are given below. 
Theorem 1.1.1. If $G$ is a planar graph, then the following hold.

(i) If $\Delta(G) \leq 4$, then $\chi_{L, 2}(G) \leq 5$;

(ii) $\chi_{L, 2}(G) \leq 6$;

(iii) $\chi_{2}(G) \leq 5$.

Theorem 1.1.2. If $G$ is a graph with genus $g(G) \geq 1$, then $\chi_{L, 2}(G) \leq \frac{1}{2}(7+\sqrt{1+48 g(G)})$.

A graph $G$ has a graph $H$ as minor if $H$ can be obtained from a subgraph of $G$ by contracting edges, and $G$ is called $H$-minor free if $G$ does not have $H$ as a minor. A graph $G$ is called a series-parallel graph if each minimal component can be obtained from $K_{2}$ by iteratively using the following two operations: replace an edge with a path of length 2 and duplicate an edge. A graph $G$ is $K_{4}$-minor free if and only if each block of $G$ is a series-parallel graph. Wegner [46] conjectured that if $G$ is a planar graph, then

$$
\chi_{\Delta}(G)= \begin{cases}\Delta(G)+5, & \text { if } 4 \leq \Delta(G) \leq 7 ; \\ \lfloor 3 \Delta(G) / 2\rfloor+1, & \text { if } \Delta(G) \geq 8 .\end{cases}
$$

Define

$$
K(r)= \begin{cases}r+3, & \text { if } 2 \leq r \leq 3 \\ \lfloor 3 r / 2\rfloor+1, & \text { if } r \geq 4\end{cases}
$$

Lih et. al. proved the following towards Wegner's conjecture.

Theorem 1.1.3. (K-W. Lih, W.-F. Wang and X. Zhu [22]) Let $G$ be a $K_{4}$-minor free graph. Then

$$
\chi_{\Delta}(G) \leq K(\Delta(G))
$$

Here, we will extend Theorem 1.1.3 as the following.

Theorem 1.1.4. Let $G$ be a $K_{4}$-minor free graph with $\Delta=\Delta(G)$, and $r \geq 2$ be an integer. Then

(i) $\chi_{r}(G) \leq K(r)$.

(ii) $\chi_{L, r}(G) \leq K(r)+1$.

Examples given in [22] show that Theorem 1.1.4 (i) is best possible when $r=\Delta$.

\subsection{Backgrounds of receptor clustering}

The receptors discussed here are membrane-bound proteins. They are kept in the membrane by electrostatic forces but can diffuse along its surface in two dimensions. The membrane has a number of features, including elements of the cytoskeleton (aka membrane skeleton), lipid rafts (special types of lipids that form aggregates are insoluble in certain detergents), caveloae (cavelike indentations formed by a lipid called caveolin) and protein agglomerations. These features 
form a "landscape" that generally inhibits the free movement or normal diffusion of membrane proteins. Instead, receptors perform anomalous diffusion characterized by a variable effective diffusion constant.

The vast majority of our current knowledge regarding the movement and localization of membrane proteins comes from innovative labeling and imaging techniques $[38,48]$ that emerged in the past couple of decades. In general, the proteins or lipids of interest are not distinguishable from the rest of the cell; therefore, imaging hinges on the ability to label these biomolecules with a tag that is clearly identifiable in the microscopic image. Important techniques used currently rely on fluorescent labels or metallic beads.

Our research is motivated by the following hypothesis. The growth of new blood vessels from preexisting vessels, is switched on or off by the dynamic balance among numerous angiogenic stimulators and inhibitors (the 'angiogenesis switch' hypothesis) [6, 19]. Among the various growth factors, vascular endothelial growth factor (VEGF) and its receptors (VEGFR) have received much attention, because of their fundamental role in tumorigenesis and other pathologies $[6,23,32]$. Initially identified as a vascular permeability factor that increased leakiness of blood vessels [42], the role of VEGF in regulating angiogenesis was discovered later $[14,36]$.

Signaling by VEGFR is initiated by binding of the ligand dimer to the extracellular domain of the receptor, which stimulates receptor homo- and hetero-dimerization [29,40,44]. Receptor dimerization is followed by protein kinase activation, trans-autophosphorylation, recruitment of signaling molecules, and activation of distinct pathways. Due to its bivalence, VEGF binding may precede and induce the dimerization of its receptors, by the binding of a second receptor to the free binding site of the ligand (see Figure 1.1 for the explicit process). Ligand-induced or -enhanced receptor dimerization is a feature present in several other receptor-ligand families including EGF and immune receptors.

Mathematical models of VEGF binding [16] generally represent the cell membrane as a single, homogeneous entity, equivalent to a "well-mixed compartment" whose state is sufficiently characterized by a single concentration value for each of the substances of interest. This is justified if there are no significant inhomogeneities and all molecules can diffuse and mix freely over the entire membrane surface, as in the classic Singer-Nicholson fluid mosaic model [43]. However, our understanding of the cell membrane has evolved significantly since 1972. The current picture [45] is more structured, with microdomains of lipids and proteins [17, 27, 41]. Modern microscopy techniques $[38,48]$ provide direct evidence of the effect of these structures on membrane receptor localization and movement [3,31,34], revealing receptor clusters in static images, and intervals of confinement in small areas separated by jumps or "hops" in single particle tracking.

Spatial organization in the membrane can potentially have a major impact on signaling pathways that rely on interaction between membrane-bound molecules. Receptor dimerization, either 


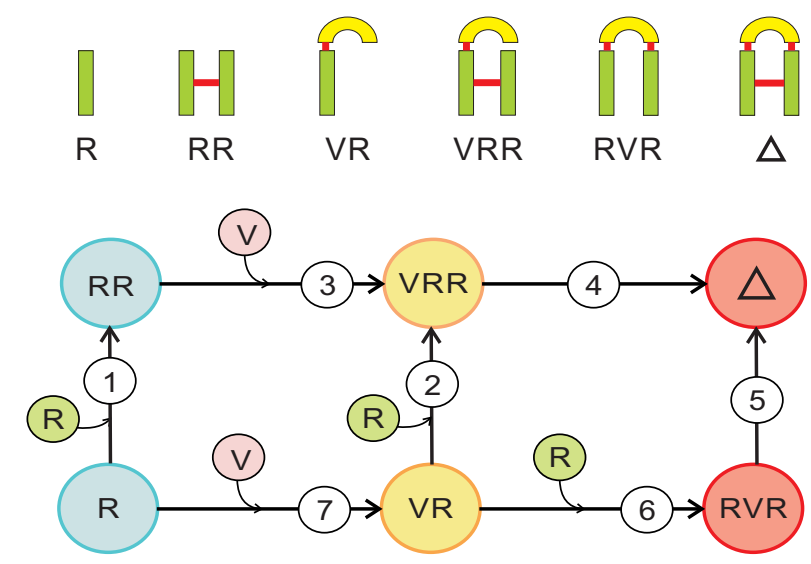

Figure 1.1: The dimerization and ligand binding reactions form a network of 7 reactions in the VEGF signal initiation model of [16]. Receptors $(R)$ may bind one of the two poles of a VEGF ligand $(V)$, and may form a direct bond with another receptor. In the ligand-induced dimerization (LID) sequence, receptors can not form a direct bond outside a pre-existing complex; signal initiation progresses through reactions $(7,6,5)$. In the dynamic pre-dimerization (DPD) sequence, receptors may dimerize before binding ligand (adding reactions 1 and 2).

through (ligand-dependent or independent) direct receptor-receptor binding, or by crosslinking through ligand $[\mathrm{s}]$, requires the collision of two membrane-bound receptors, and is thus influenced by the mobility and possible confinement of receptors. In turn, receptor dimerization is a necessary step in signal initiation, and therefore the mobility and spatial organization of membrane receptors must be part of the quantitative understanding of many cell signaling pathways. 


\section{Chapter 2}

\section{2-hued Coloring for Planar Graphs and Graphs of Higher Genus}

\subsection{Priliminaries}

A plane graph is a planar graph that is embedded in the plane. Let $G$ be a connected plane graph, and let $F$ be a face of $G$. Then the boundary of $F$ is the boundary of the open set in the usual topological sense, and it contains the vertices and edges that are incident with $F$. The degree of $F$ is the number of edges incident with $F$. We call the face with degree $k$ a $k$-face.

For a given edge $e=v_{1} v_{2}$ of $G$, let $d_{1}, d_{2}$ denote the degrees of the two endpoints $v_{1}$ and $v_{2}$ of $e$, and $d_{1}^{*}, d_{2}^{*}$ denote the degrees of the two faces adjacent at $e$, respectively. The edge contribution of $e$ is defined to be $\Phi(e)=\frac{1}{d_{1}}+\frac{1}{d_{2}}+\frac{1}{d_{1}^{*}}+\frac{1}{d_{2}^{*}}-1$. The next result is known as a Lebesgue's formulae.

Lemma 2.1.1. (Page 55 in [33]) Let $G$ be a plane graph, then $\sum_{e \in E(G)} \Phi(e)=2$.

Throughout this paper, for an edge $e$ of a plane graph $G$, we shall represent the edge configuration of $e$ as the 4 -tuple $\left(x_{1}, x_{2}, x_{3}, x_{4}\right)$ such that $x_{1} \leq x_{2} \leq x_{3} \leq x_{4}$, where $\left\{x_{1}, x_{2}, x_{3}, x_{4}\right\}=$ $\left\{d_{1}, d_{2}, d_{1}^{*}, d_{2}^{*}\right\}$ as multisets. For convenience, we use $\left(x_{1}, x_{2}, x_{3}, S\right)$ with $S$ being a set of integers, to mean that in this configuration, $x_{4}$ can be any integer in $S$. If $S$ is given by an interval (such in Lemma 2.1.2), then $S$ is the set of the integers inside the interval.

Lemma 2.1.2. Let $G$ be a plane graph with $\delta(G) \geq 3$. Then there must be an edge with its configuration falling into one of the following categories.

(i) $(3,3,3,[3, \infty))$;

(ii) $(3,3,4,[4,11])$;

(iii) $(3,3,5,[5,7])$;

(iv) $(3,4,4,[4,5])$; 
Proof. We may assume that $G$ is connected. By Lemma 2.1.1, $\sum_{e \in E(G)} \Phi(e)=2>0$, and so $G$ has an edge $e$ with $\Phi(e)>0$. We denote the configuration of $e$ by $\left(x_{1}, x_{2}, x_{3}, x_{4}\right)$. Then $\sum_{i=1}^{4} \frac{1}{x_{i}}>1$.

Since $\delta(G) \geq 3$, we have $x_{i} \geq 3$, for each $i \in\{1,2,3,4\}$. As $x_{1} \leq x_{2} \leq x_{3} \leq x_{4}, 4 \cdot \frac{1}{x_{1}}>1$, and so $x_{1}<4$. This implies that $x_{1}=3$. Thus $\sum_{i=2}^{4} \frac{1}{x_{i}}>1-\frac{1}{3}=\frac{2}{3}$. As $3 \cdot \frac{1}{5}<\frac{2}{3}$, thus $x_{2}<5$, it follows that $x_{2}=3$ or $x_{2}=4$.

If $x_{2}=3$, then $\frac{1}{x_{3}}+\frac{1}{x_{4}}>\frac{1}{3}$, hence $x_{3}<6$. It is routine to verify that if $x_{3}=3$, then $x_{4}$ can be any number no less than 3 ; if $x_{3}=4$, then $4 \leq x_{4} \leq 11$; and if $x_{3}=5$, then $5 \leq x_{4} \leq 7$.

If $x_{2}=4$, then $\frac{1}{x_{3}}+\frac{1}{x_{4}}>\frac{5}{12}$, and so $x_{3}<5$. Hence $x_{3}=4$ and $x_{4} \leq 5$. This completes the proof of the lemma.

By Lemma 2.1.2, the following properties on the local structure of a plane graph can be obtained.

Lemma 2.1.3. Let $G$ be a plane graph with $\delta(G) \geq 3$. Then there must be an edge $e=v_{1} v_{2}$ which meets at least one of the following conditions.

(i) $d\left(v_{1}\right) \leq 4$ and e lies in the boundary of a 3 -face;

(ii) $d\left(v_{1}\right)=3$ and $e$ lies in the boundary of a 4 -face;

(iii) $d\left(v_{1}\right)=d\left(v_{2}\right)=3$ and $e$ is the common boundary of a 5-face and another l-face where $5 \leq l \leq 7$

(iv) $d\left(v_{1}\right)=5,5 \leq d\left(v_{2}\right) \leq 7$ and $e$ is the common boundary of two 3-faces.

Proof. By Lemma 2.1.2, $G$ has an edge $e=v_{1} v_{2}$ satisfying the conclusion of Lemma 2.1.2. The conclusions of this lemma will follow by analyzing the four cases listed in Lemma 2.1.2.

Lemma 2.1.4. Let $G$ be a smallest counterexample to Theorem 1.1.1. Then $G$ must be connected and $\delta(G) \geq 3$.

Proof. We argue by contradiction and assume that

$$
G \text { is a counterexample with }|V(G)| \text { minimized. }
$$

Then for some list assignment $\{L(v): v \in V(G)\}, G$ has no $(L, 2)$-coloring. Furthermore, for one such list assignment $L$ and any $v \in V(G),|L(v)|=5$ if (i) does not hold for $G ;|L(v)|=6$ if (ii) does not hold for $G$; $L(v)=\{1,2,3,4,5\}$ if (iii) does not hold for $G$. By (2.1), $G$ must be connected with $|V(G)| \geq 6$.

If $\delta(G)=1$, then let $v$ be a vertex of degree 1 in $G$ and $w$ be the only neighbor of $v$. Denote $G^{\prime}=G-v$. By $(2.1), G^{\prime}$ has an $(L, 2)$-coloring $c$. Extending $c$ by coloring $v$ with $c(v) \in L(v) \backslash c\left(\left\{w, w^{\prime}\right\}\right)$, where $w^{\prime}$ is another neighbor of $w$. Then $c$ can be extended to an $(L, 2)$-coloring for $G$, contrary to $(2.1)$. 
Now suppose that $\delta(G) \geq 2$ and $v$ is a vertex of degree 2. Denote the neighbors of $v$ as $x, y$. Let $x^{\prime}, y^{\prime}$ be neighbors of $x, y$ in $G-v$, respectively. By (2.1), $G^{\prime}=G-v+x y$ has an $(L, 2)$-coloring $c$ with $c(x) \neq c(y)$. Extending $c$ by coloring $v$ with $c(v) \in L(v) \backslash c\left(\{x, y\} \cup\left\{x^{\prime}, y^{\prime}\right\}\right)$. Then the extended $c$ is an $(L, 2)$-coloring of $G$, contrary to $(2.1)$. So we must have $\delta(G) \geq 3$.

\section{$2.2 \quad$ 2-hued coloring for planar graphs}

Arguing by contradiction, we assume that

$$
G \text { is a counterexample to Theorem } 1.1 .1 \text { with }|V(G)| \text { minimized. }
$$

Then for some list assignment $\{L(v): v \in V(G)\}, G$ has no $(L, 2)$-coloring. Equivalently, we may assume that for every $v \in V(G)$,

$$
\begin{aligned}
|L(v)| & =5, \text { if (i) does not hold for } \mathrm{G} ; \\
|L(v)| & =6 \text {, if (ii) does not hold for } \mathrm{G} ; \\
L(v) & =5 \text {, if (iii) does not hold for } \mathrm{G} .
\end{aligned}
$$

By Lemma 2.1.4, $G$ must be connected with $\delta(G) \geq 3$. In the arguments below, we start with a plane graph $G^{\prime}$ with $\left|V\left(G^{\prime}\right)\right|<|V(G)|$. Then by (2.2), $G^{\prime}$ has an $(L, 2)$-coloring $c$. To obtain a contradiction, we extend the $(L, 2)$-coloring $c$ on $G^{\prime}$ to one on $G$. In the following arguments, for all unmentioned vertices $w$ in $G^{\prime}, c(w)$ will not be changed in the extension. Throughout this section, let $e=v_{1} v_{2}$ denote an edge satisfying one of (i)-(iv) in Lemma 2.1.3. By Lemma 2.1.3, one of the following four cases must occur.

Case $1 d\left(v_{1}\right) \leq 4$ and $e$ lies in the boundary of a 3 -face.

Let $G^{\prime}=G-v_{1}$. By $(2.2), G^{\prime}$ has an $(L, 2)$-coloring $c$. Extending $c$ by coloring $v_{1}$ with $c\left(v_{1}\right) \in L\left(v_{1}\right) \backslash c\left(N\left(v_{1}\right)\right)$. As $\delta\left(G^{\prime}\right) \geq 2, v_{1}$ has a pair of adjacent vertices in the 3 -face, and so the neighborhood of every vertex has at least 2 different colors. Hence $c$ is an $(L, 2)$-coloring of $G$, contrary to $(2.2)$.

Case $2 d\left(v_{1}\right)=3$ and $e$ lies in the boundary of a 4 -face.

Let $F_{1}=v_{1} v_{2} x_{1} x_{2}$ denote the boundary of this 4-face. Let $G^{\prime}=G-v_{1}+x_{2} v_{2}$. By $(2.2), G^{\prime}$ has an $(L, 2)$-coloring $c$. Extending $c$ by coloring $v_{1}$ with $c\left(v_{1}\right) \in L\left(v_{1}\right) \backslash c\left(N\left(v_{1}\right) \cup\left\{x_{1}\right\}\right)$. As $c$ is an $(L, 2)$-coloring of $G^{\prime}, c\left(x_{2}\right) \neq c\left(v_{2}\right)$. The choice of $c\left(v_{1}\right)$ makes $c$ satisfying both $(\mathrm{C} 1)$ and 
(C2). And so $c$ is an $(L, 2)$-coloring of $G$, contrary to $(2.2)$.

Case $3 d\left(v_{1}\right)=d\left(v_{2}\right)=3$ and $e$ is the common boundary of a 5 -face and an $l$-face where $5 \leq l \leq 7$.

Let $F_{1}$ denote the 5 -face, and $F_{2}$ the $l$-face. For $i=1,2$, let $x_{i}$ be the neighbor of $v_{i}$ on the boundary of $F_{1}, y_{i}$ be the neighbor of $v_{i}$ on the boundary of $F_{2}$. Thus $N\left(v_{1}\right)=\left\{x_{1}, y_{1}, v_{2}\right\}$ and $N\left(v_{2}\right)=\left\{x_{2}, y_{2}, v_{1}\right\}$. Let $G^{\prime}=G-v_{1}-v_{2}$. By $(2.2), G^{\prime}$ has an $(L, 2)$-coloring $c$. Extending $c$ by coloring $v_{1}$ with $c\left(v_{1}\right)$ from $L\left(v_{1}\right) \backslash c\left(\left\{x_{1}, y_{1}, x_{2}\right\}\right)$ and $c\left(v_{2}\right)$ from $L\left(v_{2}\right) \backslash c\left(\left\{x_{2}, y_{2}, x_{1}, v_{1}\right\}\right)$ respectively. As $c$ is an $(L, 2)$-coloring of $G^{\prime}$, and by the choice of $c\left(v_{1}\right)$ and $c\left(v_{2}\right)$, the extended $c$ satisfies both $(\mathrm{C} 1)$ and $(\mathrm{C} 2)$, and so $c$ is an $(L, 2)$-coloring of $G$, contrary to $(2.2)$.

Case $4 d\left(v_{1}\right)=5,5 \leq d\left(v_{2}\right) \leq 7$ and $e$ is the common boundary of two 3 -faces. (This case is not applicable for Theorem 1.1.1(i).)

Suppose that Theorem 1.1.1(ii) does not hold. By $(2.4),|L(v)|=6$, for all $v \in V(G)$. Let $G^{\prime}=G-v_{1}$. By $(2.2), G^{\prime}$ has an $(L, 2)$-coloring $c$. Since $d\left(v_{1}\right)=5$ in $G, L\left(v_{1}\right) \backslash c\left(N_{G}\left(v_{1}\right)\right) \neq \emptyset$. Extending $c$ by coloring $v_{1}$ with $c\left(v_{1}\right) \in L\left(v_{1}\right) \backslash c\left(N\left(v_{1}\right)\right)$. Since $e$ lies in a 3 -face, $N_{G}\left(v_{1}\right)$ contains an edge, and so $\left|c\left(N\left(v_{1}\right)\right)\right| \geq 2$. By the definition of $c\left(v_{1}\right)$ and by the assumption that $c$ is an $(L, 2)$-coloring of $G^{\prime}$, the extended $c$ is an $(L, 2)$-coloring of $G$, contrary to (2.2).

Suppose that Theorem 1.1.1(iii) does not hold. By $(2.5), L(v)=\overline{5}$, for all $v \in V(G)$. Denote the two faces as $F_{1}=v_{1} v_{2} w_{1}$ and $F_{2}=v_{1} v_{2} w_{2}$, respectively. Two subcases are discussed below.

Subcase $4.1 w_{1} w_{2} \notin E(G)$.

We obtain $G^{\prime}$ from $G-v_{1}$ by identifying $w_{1}$ with $w_{2}$ (denoting the new vertex by $w$ ). Let $L(w)=\overline{5}$. As $w_{1}$ and $w_{2}$ are in the same face of $G-v_{1}, G^{\prime}$ is again planar. By $(2.2), G^{\prime}$ has an $(L, 2)$-coloring $c$, which can also be viewed as an $(L, 2)$-coloring of $G-v_{1}$ with $w_{1}, w_{2}$ receiving the same color. Since $w_{1}$ and $w_{2}$ are identified in $G^{\prime},\left|c\left(N_{G}\left(v_{1}\right)\right)\right| \leq d_{G}\left(v_{1}\right)-1=4$, and so $L\left(v_{1}\right) \backslash c\left(N\left(v_{1}\right)\right) \neq \emptyset$. Extending $c$ by coloring $v_{1}$ with $c\left(v_{1}\right) \in L\left(v_{1}\right) \backslash c\left(N\left(v_{1}\right)\right)$. By the definition of $c\left(v_{1}\right)$ and by the assumption that $c$ is an $(L, 2)$-coloring of $G-v_{1}$, the extended $c$ is an $(L, 2)$-coloring of $G$, contrary to $(2.2)$.

Subcase $4.2 w_{1} w_{2} \in E(G)$.

For a plane graph $G$ with a cycle $C$, let $\operatorname{Ext}[C]$ (resp. Int $[C]$ ) be the subgraph obtained from $G$ by deleting all vertices inside (resp. outside) the cycle $C$. If $V(\operatorname{Ext}[C])-V(C) \neq \emptyset$ and $V(\operatorname{Int}[C])-V(C) \neq \emptyset$, then $C$ is called a separating cycle of $G$.

Note that the two faces $F_{1}$ and $F_{2}$ must be contained in one of the 3 -cycles, $v_{1} w_{1} w_{2}$ or $v_{2} w_{1} w_{2}$. 


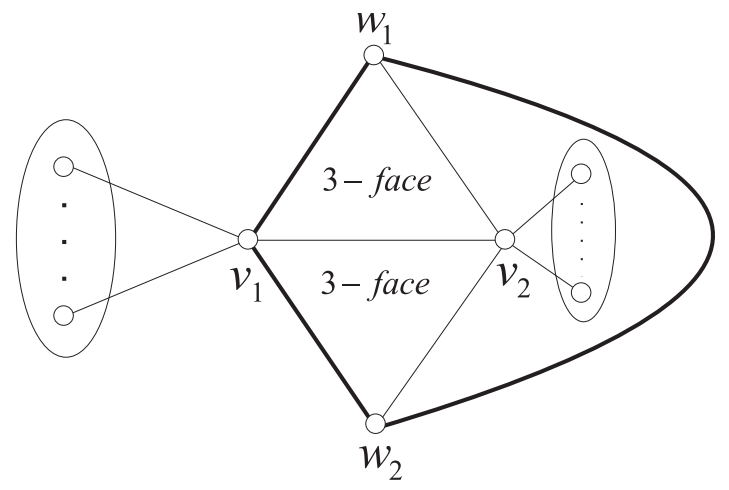

Figure 2.1: Graph for Subcase 4.2.

Without loss of generality, assume that $C=v_{1} w_{1} w_{2}$ that contains both $F_{i}$ with $i=1$, 2, see Figure 2.1. Since both $d_{G}\left(v_{i}\right) \geq 5$ with $i=1,2, C$ must be a separating cycle of $G$, and so each of $\operatorname{Ext}[C]$ and $\operatorname{Int}[C]$ has fewer vertices than $G$.

By (2.2), each of $\operatorname{Ext}[C]$ and $\operatorname{Int}[C]$ has an $(L, 2)$-coloring, denoted as $c_{1}$ and $c_{2}$, respectively. Since $G\left[v_{1}, w_{1}, w_{2}\right] \cong K_{3}$, we may assume that $c_{1}\left(v_{1}\right)=c_{2}\left(v_{1}\right), c_{1}\left(w_{1}\right)=c_{2}\left(w_{1}\right), c_{1}\left(w_{2}\right)=c_{2}\left(w_{2}\right)$.

Since $V(G)=V(\operatorname{Ext}[C]) \cup V(\operatorname{Int}[C])$ and $V(\operatorname{Ext}[C]) \cap V(\operatorname{Int}[C])=\left\{v_{1}, w_{1}, w_{2}\right\}$, and since $c_{1}$ and $c_{2}$ agree on $\left\{v_{1}, w_{1}, w_{2}\right\}$, one can construct an $(L, 2)$-coloring $c$ of $G$ by combining $c_{1}$ and $c_{2}$ :

$$
c(v)= \begin{cases}c_{1}(v), & \text { if } z \in V(\operatorname{Ext}[C]) ; \\ c_{2}(v), & \text { if } z \in V(\operatorname{Inc}[C]) .\end{cases}
$$

As $c_{1}$ and $c_{2}$ are $(L, 2)$-colorings of $\operatorname{Ext}[C]$ and $\operatorname{Int}[C]$, respectively, and as $G\left[v_{1}, w_{1}, w_{2}\right] \cong K_{3}$, $c$ is an $(L, 2)$-coloring for $G$, contrary to (2.2). This completes the proof of Theorem 1.1.

As shown in [26], $C_{5}$ is planar with $\chi_{2}\left(C_{5}\right)=5$. It follows by (1.3) that Theorem 1.1.1(i) and (iii) are best possible. We conjecture that $C_{5}$ is the only connected planar graph $G$ with $\chi_{2}(G)=5$.

When $r>2$, the $r$-hued chromatic number $\chi_{r}(G)$ of a planar graph $G$ may be larger than 5. For example, the wheel $W_{6}$ with six vertices has $\chi_{3}\left(W_{6}\right)=6$, because any pair of vertices of degree 3 that are not adjacent are adjacent to a common vertex of degree 3 , and the unique vertex of degree 5 is adjacent to all other vertices. In fact Lai et al. [25] showed that $\chi_{r}(T)=\min \{r, \Delta(T)\}+1$ if $T$ is a tree with $|V(T)| \geq 3$. Hence $\chi_{5}(T)>5$ if $\Delta(T) \geq 5$. 


\section{$2.3 \quad$ 2-hued coloring for graphs of higher genus}

An embedding of a graph $G$ on an orientable surface (resp. non-orientable surface) $\Sigma$ is minimal if $G$ cannot be embedded on any orientable (resp. non-orientable) surface $\Sigma^{\prime}$ where $g\left(\Sigma^{\prime}\right)<g(\Sigma)$. A graph $G$ is said to have orientable (resp. non-orientable) genus $g$ if $G$ is minimally embedded on a surface with orientable (resp. non-orientable) genus $g$. An embedding of a graph is said to be 2-cell if every face of the embedding is homomorphic to an open unit disk. The Euler characteristic of a graph $G$ is defined as follows.

$$
\Phi(G)= \begin{cases}2-2 g, & \text { if } G \text { has the orientable genus } g \\ 2-g, & \text { if } G \text { has the non-orientable genus } g .\end{cases}
$$

If $G$ is a connected graph with a 2-cell embedding on a closed surface, then Euler formula indicates that

$$
|V(G)|-|E(G)|+|F(G)|=\Phi(G) .
$$

The following results are needed in our proofs.

Theorem 2.3.1. ([49]) If a connected graph $G$ is minimally embedded on an orientable surface, then the embedding is 2-cell.

Theorem 2.3.2. ([35]) If $G$ is a connected graph, which is not a tree, then $G$ has a minimal non-orientable embedding which is 2-cell.

Throughout this section, we assume that $G$ is 2-cell embedded on a closed surface. Recall the edge contribution of an edge $e$ is $\Phi(e)=\frac{1}{d_{1}}+\frac{1}{d_{2}}+\frac{1}{d_{1}^{*}}+\frac{1}{d_{2}^{*}}-1$. For convenience, let $\Phi^{\prime}(e)=-\Phi(e)$.

Lemma 2.3.3 below follows from Theorems 2.3.1, 2.3.2, with a similar argument in Chapter 4 of [33], where the case $g=0$ is considered.

Lemma 2.3.3. If a connected graph $G$ is minimally embedded on a closed surface then

$$
\sum_{e \in E(G)} \Phi(e)=\Phi(G)
$$

Proof of Theorem 1.1.2. Let $g(G)$ denote the genus of $G$ and $h(G)=\frac{1}{2}(7+\sqrt{1+48 g(G)})$. By contradiction, we assume that

$$
G \text { is a counterexample to Theorem } 1.1 .2 \text { with }|V(G)| \text { minimized. }
$$

Then $g(G) \geq 1, \chi L, 2(G)>h(G)$, and $G$ has an assignment $\{L(v): v \in V(G)\}$ with $|L(v)|=$ $h(G), \forall v \in V(G)$, such that $G$ has no $(L, 2)$-coloring. By $(2.7), G$ must be connected. We establish each of the following claims. The first claim is an observation following immediately from the definition of $(L, 2)$-colorings. 
Claim 1. $|V(G)| \geq h(G)+1$.

Claim 2. $\delta(G) \geq h(G)-2$.

We prove $\delta(G) \geq 3$ first. Let $v$ be a vertex with $d_{G}(v)=\delta(G)$. If $d_{G}(v)=1$, let $N_{G}(v)=\{w\}$, $w^{\prime} \in N_{G}(w)-\{v\}$ and $G^{\prime}=G-v$. By (2.7), $\chi L, 2\left(G^{\prime}\right) \leq h\left(G^{\prime}\right)$. By the definition of genus, $g\left(G^{\prime}\right) \leq g(G)$, and so $\chi L, 2\left(G^{\prime}\right) \leq h\left(G^{\prime}\right) \leq h(G)$. Thus any $(L, 2)$-coloring $c$ of $G^{\prime}$ can be extended to an $(L, 2)$-coloring of $G$ by coloring $v$ with $c(v) \in L(v) \backslash c\left(\left\{w, w^{\prime}\right\}\right)$, contrary to (2.7).

If $d_{G}(v)=2$, denote $N_{G}(v)=\{x, y\}$, and let $x^{\prime}$ (resp. $y^{\prime}$ ) be a neighbor of $x$ (resp. $y$ ) other than $v$. Let $G^{\prime}=G-v+x y$. As $G$ is 2-cell embedded on a surface with $x$ and $y$ on the same face of $G-v$, by the dentition of genus, $g\left(G^{\prime}\right) \leq g(G)$. Hence $\chi L, 2\left(G^{\prime}\right) \leq h\left(G^{\prime}\right) \leq h(G)$. By (2.7), $G^{\prime}$ has an $(L, 2)$-coloring $c$. As $g(G) \geq 1, h(G)>5$. Hence we can extend $c$ by coloring $v$ with $c(v) \in L(v) \backslash c\left(\left\{x, y, x^{\prime}, y^{\prime}\right\}\right)$. As $c$ is an $(L, 2)$-coloring of $G^{\prime}$ and by the choice of $c(v), c$ is an $(L, 2)$-coloring of $G$, contrary to $(2.7)$.

Hence $\delta(G) \geq 3$. We argue by contradiction to prove Claim 2. Assume that $G$ has a vertex $v$ with $d_{G}(v) \leq h(G)-3$. As $\delta(G) \geq 3, \exists x, y \in N_{G}(v)$ with $x \neq y$. Let $G^{\prime}=G-v+x y$. With the same argument above, $g\left(G^{\prime}\right) \leq g(G)$. Hence $\chi L, 2\left(G^{\prime}\right) \leq h\left(G^{\prime}\right) \leq h(G)$. By (2.7), $G^{\prime}$ has an $(L, 2)$-coloring $c$. Let $x^{\prime}, y^{\prime}$ be a neighbor of $x, y$ in $G-v$, respectively. Extending $c$ by coloring $v$ with $c(v) \in L(v) \backslash c\left(N(v) \cup\left\{x^{\prime}, y^{\prime}\right\}\right)$. Since $x, y$ are adjacent in $G^{\prime}, c(x) \neq c(y)$. Since $\delta(G) \geq 3$, $\delta\left(G^{\prime}\right) \geq 2$, and so the extended $c$ violates (2.7). This proves Claim 2 .

Claim 3. Let $e=v_{1} v_{2}$ be an edge in $G$. Then either $d_{1} \geq h(G)$ or $d_{2} \geq h(G)$.

We assume otherwise that $d_{i}=d_{G}\left(v_{i}\right) \leq h(G)-1, i=1,2$. Denote $G^{\prime}=G-v_{1}-v_{2}$. By (2.7), $G^{\prime}$ has an $(L, 2)$-coloring $c$. Denote $N_{1}=N_{G}\left(v_{1}\right) \backslash\left\{v_{2}\right\}, N_{2}=N_{G}\left(v_{2}\right) \backslash\left\{v_{1}\right\}$. Then $\max \left\{\left|N_{1}\right|,\left|N_{2}\right|\right\} \leq h(G)-2$. If $\min \left\{\left|c\left(N_{1}\right)\right|,\left|c\left(N_{2}\right)\right|\right\} \geq 2$, then extend $c$ by coloring $v_{1}$ with $c\left(v_{1}\right) \in L\left(v_{1}\right) \backslash c\left(N_{1}\right)$ and $v_{2}$ with $c\left(v_{2}\right) \in L\left(v_{2}\right) \backslash c\left(\left\{N_{2} \cup v_{1}\right\}\right)$. As $c$ is an $(L, 2)$-coloring of $G^{\prime}$ and by the choices of $c\left(v_{1}\right)$ and $c\left(v_{2}\right), c$ is an $(L, 2)$-coloring of $G$, contrary to $(2.7)$.

Thus we assume that $\left|c\left(N_{2}\right)\right|=1$. Then pick $v_{1}^{\prime} \in N_{G}\left(v_{1}\right)-\left\{v_{2}\right\}$. Extending $c$ by coloring $v_{1}$ with $c\left(v_{1}\right) \in L\left(v_{1}\right) \backslash c\left(N_{1} \cup N_{2}\right)$ and $v_{2}$ with $c\left(v_{2}\right) \in L\left(v_{2}\right) \backslash c\left(\left\{N_{2} \cup\left\{v_{1}, v_{1}^{\prime}\right\}\right\}\right)$. As $c$ is an $(L, 2)$-coloring of $G^{\prime}$ and by the choices of $c\left(v_{1}\right)$ and $c\left(v_{2}\right), c$ is an $(L, 2)$-coloring of $G$, contrary to (2.7). This proves Claim 3.

Claim 4. Let $e=v_{1} v_{2}$ be an edge in $G$. If $3 \in\left\{d_{1}^{*}, d_{2}^{*}\right\}$, then $d_{i} \geq h(G), i=1,2$.

If not, we assume that $d_{1} \leq h(G)-1$. Let $G^{\prime}=G-v_{1}$. Then $g\left(G^{\prime}\right) \leq g(G)$, and so by $(2.7)$, $G^{\prime}$ has an $(L, 2)$-coloring $c$. Extending $c$ by coloring $v_{1}$ with $c\left(v_{1}\right) \in L\left(v_{1}\right) \backslash c\left(N\left(v_{1}\right)\right)$. As $c$ is an $(L, 2)$-coloring of $G^{\prime}$ and by the choices of $c\left(v_{1}\right), c$ is an $(L, 2)$-coloring of $G$, contrary to $(2.7)$. This proves Claim 4. 
Claim 5. Let $e=v_{1} v_{2}$ be an edge in $G$. If $4 \in\left\{d_{1}^{*}, d_{2}^{*}\right\}$, then $d_{i} \geq h(G)-1, i=1,2$.

If otherwise, we may assume that $d_{1}^{*}=4$ and $d_{1} \leq h(G)-2$. Denote $F=v_{1} v_{2} u w v_{1}$ as the 4-face. Let $G^{\prime}=G-v_{1}+w v_{2}$. Then by our assumption, $G^{\prime}$ has an $(L, 2)$-coloring $c$, and so $c(w) \neq c\left(v_{2}\right)$. Extending $c$ by letting $c\left(v_{1}\right) \in L\left(v_{1}\right) \backslash c\left(N\left(v_{1}\right) \cup\{u\}\right)$, contrary to the choice of $G$. This proves Claim 5.

For notational convenience, we shall denote $h(G)$ and $g(G)$ by $h$ and $g$ respectively throughout the rest of the proof.

Claim 6. Let $e=v_{1} v_{2}$ be an edge in $G$. Each of the following holds:

(i) If $3 \in\left\{d_{1}^{*}, d_{2}^{*}\right\}$, then

$$
\Phi^{\prime}(e) \geq \frac{h-6}{3 h} .
$$

(ii) If $3 \notin\left\{d_{1}^{*}, d_{2}^{*}\right\}, 4 \in\left\{d_{1}^{*}, d_{2}^{*}\right\}$, then

$$
\Phi^{\prime}(e) \geq \frac{h^{2}-5 h+2}{2 h(h-1)} .
$$

(iii) If $d_{1}^{*}, d_{2}^{*} \geq 5$, then

$$
\Phi^{\prime}(e) \geq \frac{3 h^{2}-16 h+10}{5 h(h-2)} .
$$

By Claim $2, \delta(G) \geq 3$. Thus $d_{i} \geq 3, d_{i}^{*} \geq 3, i=1,2$. If $3 \in\left\{d_{1}^{*}, d_{2}^{*}\right\}$, then by Claim $4, d_{i} \geq h$, $i=1,2$. Thus $\Phi^{\prime}(e)=1-\frac{1}{d_{1}}-\frac{1}{d_{2}}-\frac{1}{d_{1}^{*}}-\frac{1}{d_{2}^{*}} \geq 1-\frac{1}{h}-\frac{1}{h}-\frac{1}{3}-\frac{1}{3}=\frac{h-6}{3 h}$.

If $3 \notin\left\{d_{1}^{*}, d_{2}^{*}\right\}$ and $4 \in\left\{d_{1}^{*}, d_{2}^{*}\right\}$, then by Claim $5, d_{i} \geq h-1, i=1,2$. By Claim 3 , at least one of the $d_{i}$ 's must be at least $h$, and so $\Phi^{\prime}(e)=1-\frac{1}{d_{1}}-\frac{1}{d_{2}}-\frac{1}{d_{1}^{*}}-\frac{1}{d_{2}^{*}} \geq 1-\frac{1}{h-1}-\frac{1}{h}-\frac{1}{4}-\frac{1}{4}=\frac{h^{2}-5 h+2}{2 h(h-1)}$.

If $d_{1}^{*}, d_{2}^{*} \geq 5$, then by Claim $2, \delta(G) \geq h-2$. By Claim 3 , at least one of the $d_{i}$ 's must be at least $h(G)$, and so $\Phi^{\prime}(e)=1-\frac{1}{d_{1}}-\frac{1}{d_{2}}-\frac{1}{d_{1}^{*}}-\frac{1}{d_{2}^{*}} \geq 1-\frac{1}{h-2}-\frac{1}{h}-\frac{1}{5}-\frac{1}{5}=\frac{3 h^{2}-16 h+10}{5 h(h-2)}$. This proves Claim 6.

Since

$$
\frac{h-6}{3 h}<\frac{h^{2}-5 h+2}{2 h(h-1)}<\frac{3 h^{2}-16 h+10}{5 h(h-2)} .
$$

The following claim follows from Claim 6 and (2.8).

Claim 7. For each $e \in E(G)$,

$$
\Phi^{\prime}(e) \geq \frac{h-6}{3 h}
$$

Claim 8. $|E(G)| \geq \frac{1}{2}(h+3)(h-2)$.

If $\delta(G) \geq h$, by Claim 1 , we have $|V(G)| \geq h+1$, so $|E(G)| \geq \frac{1}{2}(h+1) h>\frac{1}{2}(h+3)(h-2)$. If $\delta(G)<h$, let $v$ be a vertex of $G$ such that $d(v)=\delta(G)$. Let $u$ be any neighbor of $v$, 
by Claim 3, $d(u) \geq h$. Thus there exists at least $\delta(G)$ vertices of degree at least $h$, and so $|E(G)| \geq \frac{1}{2}((h+1) \delta(G)+\delta(G)(h-\delta(G)))$. By Claim 2, $\delta \geq h-2$. When $\delta(G)=h-1$, we have that $|E(G)| \geq \frac{1}{2}(h+2)(h-1)>\frac{1}{2}(h+3)(h-2)$. When $\delta(G)=h-2$, we have that $|E(G)| \geq \frac{1}{2}(h+3)(h-2)$. This proves Claim 8 .

By Claim 2, $\delta(G) \geq h-2 \geq 5$. So $G$ is not a tree. By Theorem 2.3.1 and Theorem 2.3.2, $G$ has a 2-cell embedding. By Lemma 2.3.3, $\Phi(G)=\sum_{e \in E(G)} \Phi(e)$. Since we let $\Phi(e)=-\Phi^{\prime}(e)$, we have $-\Phi(G)=\sum_{e \in E(G)} \Phi^{\prime}(e)$. Now the rest of the proof is divided into 3 cases.

Case 1. $\delta(G) \geq h$.

By Claim 1, we have $|V(G)| \geq h+1$, so $|E(G)| \geq \frac{1}{2}(h+1) h$.

$$
\begin{aligned}
-\Phi(G) & =\sum_{e \in E(G)} \Phi^{\prime}(e) \geq \frac{1}{2} h(h+1) \cdot \frac{h-6}{3 h}=\frac{1}{24}(2 h)(2 h-10)-1 \\
& =\frac{1}{24}(7+\sqrt{1+48 g})(\sqrt{1+48 g}-3)-1=\frac{1}{24}(48 g-20+4 \sqrt{1+48 g})-1 \\
& =2 g-2+\frac{1}{6} \sqrt{1+48 g}+\frac{1}{6}>2 g-2 .
\end{aligned}
$$

Case 2. $\delta(G)=h-1$.

Let $v$ be the vertex with $d(v)=h-1$. By Claim 4, every edge $e$ incident to $v$ can not lie in a 3-face, otherwise we can deduce that $d(v) \geq h$. By Claim 6 and $(2.8), \Phi^{\prime}(e) \geq \frac{h^{2}-5 h+2}{2 h(h-1)}$ holds for every edge $e$ incident to $v$.

$$
\begin{aligned}
-\Phi(G) & =\sum_{e \in E(G)} \Phi^{\prime}(e) \geq|E(G)| \cdot \frac{h-6}{3 h}+(h-1)\left(\frac{h^{2}-5 h+2}{2 h(h-1)}-\frac{h-6}{3 h}\right) \\
& \geq \frac{1}{2}(h+3)(h-2) \cdot \frac{h-6}{3 h}+(h-1)\left(\frac{h^{2}-5 h+2}{2 h(h-1)}-\frac{h-6}{3 h}\right)=\frac{1}{6}\left(h^{2}-4 h-13\right)+\frac{5}{h} \\
& =\frac{1}{24}(2 h)(2 h-8)-\frac{13}{6}+\frac{5}{h}=\frac{1}{24}(7+\sqrt{1+48 g})(\sqrt{1+48 g}-1)-\frac{13}{6}+\frac{5}{h} \\
& =\frac{1}{24}(48 g-6+6 \sqrt{1+48 g})-\frac{13}{6}+\frac{5}{h}=2 g-2+\frac{1}{12}(3 \sqrt{1+48 g}-5)+\frac{5}{h}>2 g-2 .
\end{aligned}
$$

Case 3. $\delta(G)=h-2$.

Let $v$ be the vertex with $d(v)=h-2$. By Claim 4 and Claim 5 , every edge $e$ incident to $v$ can lie in neither a 3 -face nor a 4 -face. By Claim 6(iii), $\Phi^{\prime}(e) \geq \frac{3 h^{2}-16 h+10}{5 h(h-2)}$ holds for every edge $e$ incident to $v$. 


$$
\begin{aligned}
-\Phi(G) & =\sum_{e \in E(G)} \Phi^{\prime}(e) \geq|E(G)| \cdot \frac{h-6}{3 h}+(h-2)\left(\frac{3 h^{2}-16 h+10}{5 h(h-2)}-\frac{h-6}{3 h}\right) \\
& \geq \frac{1}{2}(h+3)(h-2) \cdot \frac{h-6}{3 h}+(h-2)\left(\frac{3 h^{2}-16 h+10}{5 h(h-2)}-\frac{h-6}{3 h}\right)=\frac{1}{30}\left(5 h^{2}-17 h-76\right)+\frac{4}{h} \\
& =\frac{1}{120}(2 h)(10 h-34)-\frac{76}{30}+\frac{4}{h}=\frac{1}{120}(7+\sqrt{1+48 g})(5 \sqrt{1+48 g}+1)-\frac{76}{30}+\frac{4}{h} \\
& =\frac{1}{120}(240 g+12+36 \sqrt{1+48 g})-\frac{76}{30}+\frac{4}{h}=2 g-2+\frac{1}{30}(9 \sqrt{1+48 g}-13)+\frac{4}{h}>2 g-2 .
\end{aligned}
$$

Thus in each case we have $-\Phi(G)>2 g-2$, contrary to (2.6). This completes the proof of Theorem 1.1.2.

The corollary below follows immediately from Theorem 1.1.2 and (1.3).

Corollary 2.3.4. If $G$ is a graph with genus $g(G) \geq 1$, then $\chi_{2}(G) \leq \frac{1}{2}(7+\sqrt{1+48 g(G)})$.

Note that a well-known result by P. Franklin [15], G. Ringel [37] and J.W.T. Youngs [49] (see also Theorem 8-8 [47]) states that, for $g(G) \geq 1, \chi(G) \leq \frac{1}{2}(7+\sqrt{1+48 g(G)})$ is indeed best possible, except for Klein bottle. By formula (1) and (3), $\chi(G) \leq \chi_{2}(G) \leq \chi L, 2(G)$. So Theorem 1.1.2 and Corollary 2.3.4 is also best possible. 


\section{Chapter 3}

\section{$r$-hued Coloring of $K_{4}$-minor Free Graphs}

\subsection{Proof of the main result}

Define $S_{G}(u)=\left\{x: d_{G}(x) \geq 3\right.$ with $u x \in E(G)$ or there exists a 2-vertex $w$ with $u w, w x \in$ $E(G)\}$. Let $D_{G}(u)=\left|S_{G}(u)\right|$. See Figure 3.1 for the case of $D_{G}(u)=2$. It is well known [11] that every $K_{4}$-minor free graph contains a vertex of degree at most two. Lih et. al. [22] proved the following lemma.

Lemma 3.1.1. (K-W. Lih, W.-F. Wang and X. Zhu [22]) Let $G$ be a $K_{4}$-minor free graph. Then one of the following conditions holds:

(i) $\delta(G) \leq 1$;

(ii) There exists two adjacent 2-vertices;

(iii) There exists a vertex $u$ with $d_{G}(u) \geq 3$ such that $D_{G}(u) \leq 2$.

We will use Lemma 3.1.1 to prove our result. Before that, we introduce some notations.

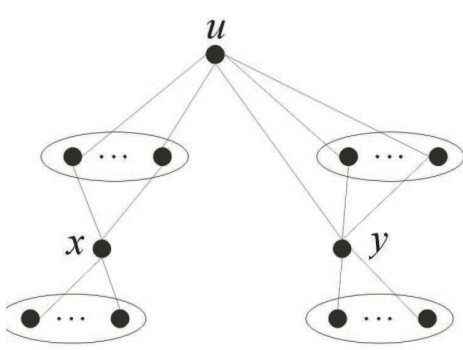

Figure 3.1: $S_{G}(u)=\{x, y\}$ 
Let $G$ be a graph with the vertex set $V, V^{\prime} \subseteq V$ be a vertex subset and $G\left[V^{\prime}\right]$ be the induced subgraph of $G$ on $V^{\prime}$. A mapping $c: V^{\prime} \rightarrow \cup_{v \in V^{\prime}} L(v)$ is a partial coloring if $c$ is a proper $(L, r)$ coloring of $G\left[V^{\prime}\right]$. Let $c$ be a partial coloring of $G$ on $V^{\prime}$. For the uncolored vertex $v \in V-V^{\prime}$, let $\{c(v)\}=\emptyset$. For every vertex $v \in V^{\prime}$, define $c[v]$ as follows.

$$
c[v]= \begin{cases}\{c(v)\}, & \text { if }\left|c\left(N_{G}(v)\right)\right| \geq r \\ \{c(v)\} \cup c\left(N_{G}(v)\right), & \text { otherwise. }\end{cases}
$$

Thus, when a partial coloring $c$ is given, $c[v]$ consists of the set of colors that can not be used for uncolored neighbors of $v$. By (3.1), $|c[v]| \leq r$.

Proof of Theorem 1.1.4. As it is shown in [8] that $\chi_{2}(G) \leq 5$ and $\chi_{L, 2}(G) \leq 6$ if $G$ is a planar graph, Theorem 1.1.4 holds for $r=2$. In the following, we assume that $r \geq 3$.

We argue by contradiction to prove Theorem 1.1.4. Assume that

$G$ is a counterexample to Theorem 1.1 .4 with $|V(G)|$ minimized.

Then for some list assignment $\{L(v): v \in V(G)\}, G$ has no $(L, r)$-coloring. We may assume that for every $v \in V(G), L(v)=\{1,2, \cdots, K(r)\}$ if $G$ is a counterexample of Theorem 1.1.4 (i), and that $|L(v)|=K(r)+1$ if $G$ is a counterexample of Theorem 1.1.4 (ii). As $r \geq 3$ implies $K(r) \geq 6$, we may assume $|V(G)| \geq 7$. By (3.2), $G$ must be connected.

In the following proof, we will obtain a $K_{4}$-minor free graph $H$ by making local modifications of $G$ such that $|V(H)|<|V(G)|$. By (3.2), $H$ has an $(L, r)$-coloring $c$. To obtain a contradiction, we shall extend and modify $c$ to an $(L, r)$-coloring of $G$.

Claim 3.1.1. $\delta(G)=2$.

If $G$ has a vertex $x$ of degree 1 , then let $H=G-x$. As $H$ is a $K_{4}$-minor free graph with $|V(H)|<|V(G)|$, it follows by (3.2) that $H$ has an $(L, r)$-coloring $c$. Let $N_{G}(x)=\{u\}$. By (3.1) and the definition of $K(r),|c[u]| \leq r<K(r)$, and so the number of colors that cannot be used for the uncolored neighbor $x$ of the vertex $u$ in $G$ is less than $K(r)$. Therefore, we can extend $c$ to an $(L, r)$-coloring of $G$ by defining $c(x) \in L(x)-c[u]$, contrary to (3.2).

Claim 3.1.2. Any two 2-vertices of $G$ are not adjacent.

If $\mathrm{G}$ has two adjacent 2-vertices $x$ and $y$, then denote $N_{G}(x)=\{u, y\}$ and $N_{G}(y)=\{v, x\}$. Let $H=G-x+u y$. As $H$ is $K_{4}$-minor with $|V(H)|<|V(G)|$, by (3.2), $H$ has an $(L, r)$-coloring $c$. For such a coloring $c$, it follows that $c[y]=\{c(y), c(v)\}$.

$$
c[u] \cap c[y]= \begin{cases}\{c(u)\}, & \text { if } c[u]=\{c(u)\} \\ \{c(u), c(y)\}, & \text { if } c[u]=\{c(u)\} \cup c\left(N_{H}(u)\right) \text { and } N_{H}(u) \cap N_{H}(y)=\emptyset . \\ \{c(u), c(y), c(w)\}, & \text { if } c[u]=\{c(u)\} \cup c\left(N_{H}(u)\right) \text { and } N_{H}(u) \cap N_{H}(y)=\{w\} .\end{cases}
$$


Therefore, $|c[u] \cup c[y]|=|c[u]|+|c[y]|-|c[u] \cap c[y]| \leq r+3-1<K(r)$, and so the number of colors that cannot be used for the uncolored neighbor $x$ of the vertices $u, y$ in $G$ is less than $K(r)$. Thus, we can extend $c$ to an $(L, r)$-coloring of $G$ by defining $c(x) \in L(x)-c[u] \cup c[y]$, contrary to $(3.2)$.

By Lemma 3.1.1, Claim 3.1.1 and Claim 3.1.2, $G$ has a vertex $u$ with $d_{G}(u) \geq 3$ such that $D_{G}(u) \leq 2$. In the rest of the proof, we always assume that $u$ is such a vertex. For $x \in S_{G}(u)$, define

$$
M_{G}(u, x)=\left\{w: w \in N_{G}(u) \cap N_{G}(x), d_{G}(w)=2\right\} \text { and } m_{G}(x)=\left|M_{G}(u, x)\right| .
$$

Without loss of generality, we may assume $m_{G}(x) \geq 1$, and we have the following claim.

Claim 3.1.3. $D_{G}(u)=2$.

By the definition of $D_{G}(u)$, Claim 3.1.1 and Claim 3.1.2, $D_{G}(u) \geq 1$. Assume that $D_{G}(u)=1$ and $S_{G}(u)=\{x\}$. Then all the neighbors of $u$ are either $x$ or some neighbors of $x$. Since $m_{G}(x) \geq 1$, pick $w \in M_{G}(u, x)$ and define $H=G-w$. As $H$ is a also $K_{4}$-minor free graph with $|V(H)|<|V(G)|$, by (3.2), $H$ has an $(L, r)$-coloring. Since $d_{G}(u) \geq 3$, we have $m_{G}(x) \geq 2$, and so $c(u) \neq c(x)$. It follows that $|c(u) \cup c[x]| \leq 1+r<K(r)$, and so the number of colors that cannot be used for $w \in M_{G}(u, x)$ is less than $K(r)$. Therefore, as $c(u) \neq c(x)$, we can extend $c$ to an $(L, r)$-coloring of $G$ by defining $c(w) \in L(w)-c[u] \cup c[x]$, contrary to (3.2).

Claim 3.1.4. Let $w \in M_{G}(u, x)$ and $c$ be an $(L, r)$-coloring of $G-w$ with $c(u) \neq c(x)$. Then $\max \left\{d_{G}(u), d_{G}(x)\right\} \leq r$.

We argue by contradiction and assume that $\max \left\{d_{G}(u), d_{G}(x)\right\}=d_{G}(u)>r$. Since $w \in$ $M_{G}(u, x)$, then $d_{G-w}(u) \geq r$. Hence by (3.1), for any $(L, r)$-coloring $c$ of $G-w,|c[u]|=1$. As $|c[u] \cup c[x]| \leq|c[u]|+|c[x]| \leq r+1<K(r)$, the number of colors that cannot be used for the uncolored $w$ in $G$ is less than $K(r)$. Therefore, by $c(u) \neq c(x), c$ can be extended to an $(L, r)$-coloring of $G$ by choosing $c(w) \in L(w)-c[u] \cup c[x]$, contrary to (3.2).

By Claim 3.1.3, $D_{G}(u)=2$. Let $S_{G}(u)=\{x, y\}$. Then by the definition of $S_{G}(u)$, it follows that (see Figure 3.1)

$$
N_{G}(u) \subseteq N_{G}(x) \cup N_{G}(y) \cup\{x, y\} .
$$

Without loss of generality, we assume that $m_{G}(x) \geq m_{G}(y)$. Since $d_{G}(u) \geq 3$, we have $m_{G}(x) \geq$ 1. Pick $w \in M_{G}(u, x)$ and define

$$
H=G-w
$$

Then $H$ is a also $K_{4}$-minor free graph with $|V(H)|<|V(G)|$. By (3.2), $H$ has an $(L, r)$-coloring.

Case 1. $x u \in E(G)$. 
As $x u \in E(H), c(u) \neq c(x)$. By Claim 3.1.4, we have $\max \left\{d_{G}(u), d_{G}(x)\right\} \leq r$. Since $x$ is adjacent to $u$, we have $|c[u] \cup c[x]| \leq d_{G}(u)+d_{G}(x)-m_{G}(x)-1$. By $m_{G}(x)+m_{G}(y) \geq d_{G}(u)-2$ and by $m_{G}(x) \geq m_{G}(y)$, we conclude that $m_{G}(x) \geq\left\lceil\left(d_{G}(u)-2\right) / 2\right\rceil=\left\lceil d_{G}(u) / 2\right\rceil-1$. Hence

$$
\begin{aligned}
|c[u] \cup c[x]| & \leq d_{G}(u)+d_{G}(x)-m_{G}(x)-1 \\
& \leq d_{G}(u)+d_{G}(x)-\left\lceil d_{G}(u) / 2\right\rceil \\
& \leq\left\lfloor d_{G}(u) / 2\right\rfloor+d_{G}(x) \\
& \leq\lfloor 3 r / 2\rfloor \\
& \leq K(r)-1 .
\end{aligned}
$$

It follows that the number of colors cannot be used for the uncolored neighbor $w$ of the vertices $u, x$ in $G$ is less than $K(r)$. Therefore, as $c(u) \neq c(x), c$ can be extended to an $(L, r)$ coloring of $G$ by choosing $c(w) \in L(w)-c[u] \cup c[x]$, contrary to (3.2). This proves Case 1 .

Case 2. $x u \notin E(G), y u \notin E(G)$.

Since $x u, y u \notin E(G)$ and $m_{G}(x) \geq m_{G}(y)$, we conclude that $m_{G}(x) \geq\left\lceil d_{G}(u) / 2\right\rceil \geq 2$. By $d_{G}(u) \geq 3$ and $m_{G}(x) \geq m_{G}(y)$, there exists a 2-vertex $w^{\prime}$ with $w^{\prime} x, w^{\prime} u \in E(H)$, and so $c(u) \neq c(x)$. By Claim 3.1.4, we have $\max \left\{d_{G}(u), d_{G}(x)\right\} \leq r$. Since $x$ is not adjacent to $u$, we have $|c[u] \cup c[x]| \leq d_{G}(u)+d_{G}(x)-m_{G}(x)+1$. Hence

$$
\begin{aligned}
|c[u] \cup c[x]| & \leq d_{G}(u)+d_{G}(x)-m_{G}(x)+1 \\
& \leq d_{G}(u)+d_{G}(x)-\left\lceil d_{G}(u) / 2\right\rceil+1 \\
& \leq\left\lfloor d_{G}(u) / 2\right\rfloor+d_{G}(x)+1 \\
& \leq\lfloor 3 r / 2\rfloor+1 \\
& \leq \begin{cases}K(3)-1 & \text { if } r=3, \\
K(r) & \text { if } r \geq 4 .\end{cases}
\end{aligned}
$$

If $|c[u] \cup c[x]|<K(r)$ (the case when $r=3$ is included), or if $|c[u] \cup c[x]|=K(r)$ and $|L(w)|=K(r)+1$, then the number of colors that cannot be used for the uncolored neighbor $w$ of the vertices $u, x$ in $G$ is less than $|L(w)|$. Therefore, as $c(u) \neq c(x)$, we can extend $c$ to an $(L, r)$-coloring of $G$ by defining $c(w) \in L(w)-c[u] \cup c[x]$, contrary to (3.2).

Therefore, we assume that $r \geq 4$ and $|c[u] \cup c[x]|=K(r)$. Since $|c[u] \cup c[x]|=\mid c\left(N_{H}(u) \cup\right.$ $\left.N_{H}(x) \cup\{u, x\}\right) \mid=K(r)$ and $N_{H}(u) \cup N_{H}(x) \cup\{u, x\}$ has exactly $K(r)$ vertices, each vertex in $N_{H}(u) \cup N_{H}(x) \cup\{u, x\}$ is colored differently by $c$. As $r \geq 4, d_{H}(u)+2 \leq(r-1)+2 \leq K(r)-2$, and so there are at least two choices to color $u$ properly in $H$ (as an $(L, r)$-coloring). By changing the color of $u$ and preserving the colors of other vertices, we obtain a new coloring 
(also denoted by $c$ ) satisfying $|c[u] \cup c[x]|<K(r)$. Therefore, as $c(u) \neq c(x)$, we can extend $c$ to an $(L, r)$-coloring of $G$ by defining $c(w) \in L(w)-c[u] \cup c[x]$, contrary to (3.2). This proves case 2 .

Case 3. $x u \notin E(G), y u \in E(G)$.

If $m_{G}(x)=m_{G}(y)$, we may interchange $x$ and $y$, and it falls under Case 1 . Hence we may assume that $m_{G}(x)>m_{G}(y)$.

Case $3.1 d_{G}(u)$ is odd.

Since $d_{G}(u)$ is odd, $m_{G}(x)+m_{G}(y)=d_{G}(u)-1$ is even, and so $m_{G}(x) \geq m_{G}(y)+2 \geq 2$.

Case 3.1.1. $m_{G}(x) \geq m_{G}(y)+4$.

Since $m_{G}(x) \geq m_{G}(y)+4 \geq 4, M_{H}(u, x) \neq \emptyset$, and so $c(u) \neq c(x)$. By Claim 3.1.4, we have $\max \left\{d_{G}(u), d_{G}(x)\right\} \leq r$. Hence,

$$
\begin{aligned}
|c[u] \cup c[x]| & \leq d_{G}(u)+d_{G}(x)-m_{G}(x)+1 \\
& \leq d_{G}(u)+d_{G}(x)-\left(d_{G}(u)+3\right) / 2+1 \\
& =\left\lfloor d_{G}(u) / 2\right\rfloor+d_{G}(x) \\
& \leq\lfloor 3 r / 2\rfloor \\
& \leq K(r)-1 .
\end{aligned}
$$

It follows that the number of colors that cannot be used for the uncolored neighbor $w$ of the vertices $u, x$ in $G$ is less than $K(r)$. Therefore, as $c(u) \neq c(x)$, we can extend $c$ to an $(L, r)$ coloring of $G$ by defining $c(w) \in L(w)-c[u] \cup c[x]$, contrary to (3.2).

Case 3.1.2. $m_{G}(x)=m_{G}(y)+2$.

If $m_{G}(x)=m_{G}(y)+2 \geq 2$, then $M_{H}(u, x) \neq \emptyset$, and so $c(u) \neq c(x)$. By Claim 3.1.4, we have $\max \left\{d_{G}(u), d_{G}(x)\right\} \leq r$. If $d_{G}(u)<r$, then

$$
\begin{aligned}
|c[u] \cup c[x]| & \leq d_{G}(u)+d_{G}(x)-m_{G}(x)+1 \\
& \leq d_{G}(u)+d_{G}(x)-\left(d_{G}(u)+1\right) / 2+1 \\
& =\left(d_{G}(u)+1\right) / 2+d_{G}(x) \\
& \leq\lfloor r / 2\rfloor+d_{G}(x) \\
& \leq\lfloor 3 r / 2\rfloor \\
& \leq K(r)-1 .
\end{aligned}
$$

Thus the number of colors that cannot be used for the uncolored neighbor $w$ of the vertices $u, x$ in $G$ is less than $K(r)$. Therefore, as $c(u) \neq c(x)$, we can extend $c$ to an $(L, r)$-coloring of $G$ by defining $c(w) \in L(w)-c[u] \cup c[x]$, contrary to $(3.2)$. 
So we assume that $d_{G}(u)=r$. If $x y \in E(G)$, then

$$
\begin{aligned}
|c[u] \cup c[x]| & \leq d_{G}(u)+d_{G}(x)-1-\left(m_{G}(x)-1\right) \\
& \leq d_{G}(u)+d_{G}(x)-\left(d_{G}(u)+1\right) / 2 \\
& =\left(d_{G}(u)-1\right) / 2+d_{G}(x) \\
& \leq\lfloor r / 2\rfloor+d_{G}(x) \\
& \leq\lfloor 3 r / 2\rfloor \\
& \leq K(r)-1 .
\end{aligned}
$$

It follows that the number of colors that cannot be used for the uncolored neighbor $w$ of the vertices $u, x$ in $G$ is less than $K(r)$. Therefore, as $c(u) \neq c(x)$, we can extend $c$ to an $(L, r)$ coloring of $G$ by defining $c(w) \in L(w)-c[u] \cup c[x]$, contrary to (3.2).

Thus we assume that $d_{G}(u)=r$ and $x y \notin E(G)$. In this case,

$$
\begin{aligned}
|c[u] \cup c[x]| & \leq d_{G}(u)+d_{G}(x)-\left(m_{G}(x)-1\right) \\
& \leq d_{G}(u)+d_{G}(x)-\left(d_{G}(u)+1\right) / 2+1 \\
& =\left(d_{G}(u)-1\right) / 2+d_{G}(x)+1 \\
& \leq\lfloor r / 2\rfloor+d_{G}(x)+1 \\
& \leq\lfloor 3 r / 2\rfloor+1 \\
& \leq \begin{cases}K(3)-1 & \text { if } r=3, \\
K(r) & \text { if } r \geq 4 .\end{cases}
\end{aligned}
$$

If $|c[u] \cup c[x]|<K(r)$ (the case when $r=3$ is included), or if $|c[u] \cup c[x]|=K(r)$ and $|L(w)|=K(r)+1$, then the number of colors that cannot be used for the uncolored neighbor $w$ of the vertices $u, x$ in $G$ is less than $|L(w)|$. Therefore, as $c(u) \neq c(x)$, we can extend $c$ to an $(L, r)$-coloring of $G$ by defining $c(w) \in L(w)-c[u] \cup c[x]$, contrary to (3.2).

Therefore, we assume that $r \geq 4$ and $|c[u] \cup c[x]|=K(r)$. Since $d_{G}(u)=d_{G}(x)=r \geq 4$ is odd, we have $d_{G}(u)=r \geq 5$. As $m_{G}(x)=m_{G}(y)+2$ and $d_{G}(u) \geq 5, M_{G}(u, y) \neq \emptyset$, and so we may choose some $w^{\prime} \in M_{G}(u, y)$. Now let $H^{\prime}=G-w-w^{\prime}+x y$ (see Figure 3.2). Then $H^{\prime}$ is also a $K_{4}$-minor free graph with $\left|V\left(H^{\prime}\right)\right|<|V(G)|$. By (3.2), $H^{\prime}$ has a $(L, r)$-coloring $c$ in which $c(u) \neq c(x)$. So we can extend $c$ to $V(G-w)$ by letting $c\left(w^{\prime}\right)=c(x)$. Then as $c(x) \in c[u]$ in $H=G-w$, the extended coloring $c$ is an $(L, r)$-coloring of $G-w$ in which $c(u) \neq c(x)$ and $|c[u] \cup c[x]|<K(r)$, and so the number of colors that cannot be used for the uncolored neighbor $w$ of the vertices $u, x$ in $G$ is less than $K(r)$. Therefore, as $c(u) \neq c(x), c$ can be further extended to an $(L, r)$-coloring of $G$ by defining $c(w) \in L(w)-c[x] \cup c[u]$, contrary to (3.2).

Case $3.2 d_{G}(u)$ is even.

If $d_{G}(u)$ is even, then $m_{G}(x)+m_{G}(y)=d_{G}(u)-1$ is odd and $m_{G}(x) \geq m_{G}(y)+1$. 

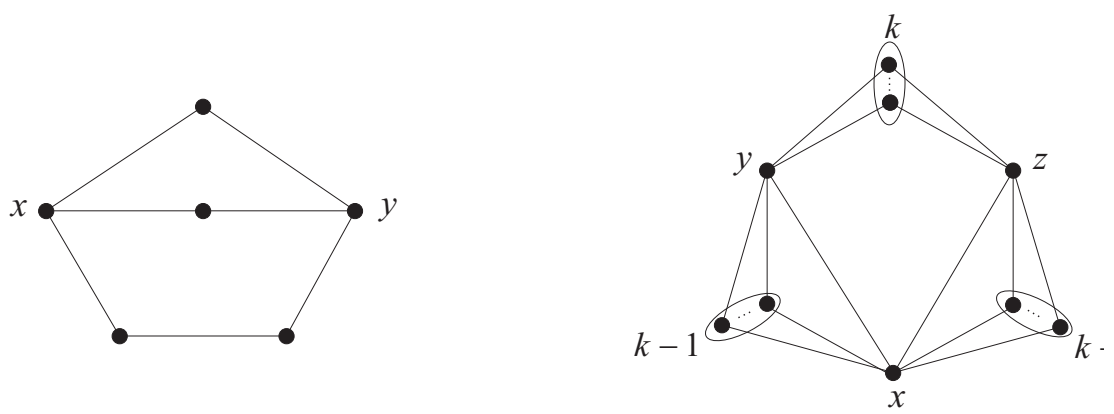

Figure 3.2: $H^{\prime}=G-w-w^{\prime}+x y$

If $m_{G}(x) \geq m_{G}(y)+3 \geq 3$, then $m_{H}(x) \geq 2$, and so $c(u) \neq c(x)$. By Claim 3.1.4, we have $\max \left\{d_{G}(u), d_{G}(x)\right\} \leq r$. Hence $|c[u] \cup c[x]| \leq d_{G}(u)+d_{G}(x)-m_{G}(x)+1 \leq d_{G}(x)+d_{G}(u) / 2$. Then

$$
|c[u] \cup c[x]| \leq \begin{cases}r+\lfloor r / 2\rfloor, & \text { if } d_{G}(u)<r ; \\ 3 r / 2, & \text { if } d_{G}(u)=r .\end{cases}
$$

Since $d_{G}(u)$ is even, when $d_{G}(u)=r, 3 r / 2=\lfloor 3 r / 2\rfloor$, and so $|c[u] \cup c[x]| \leq\lfloor 3 r / 2\rfloor \leq K(r)-1$. Then the number of colors that cannot be used for the uncolored neighbor $w$ of the vertices $u, x$ in $G$ is less than $K(r)$. Therefore, as $c(u) \neq c(x)$, we can extend $c$ to an $(L, r)$-coloring of $G$ by defining $c(w) \in L(w)-c[u] \cup c[x]$, contrary to $(3.2)$.

Assume that $m_{G}(x)=m_{G}(y)+1$. Since $d_{G}(u) \geq 4, m_{G}(y)=d_{G}(u) / 2-1 \geq 1$. Choose $w^{\prime} \in M_{G}(u, y)$ and let $H^{\prime \prime}=G-w^{\prime}$. Then $H^{\prime \prime}$ is a $K_{4}$-minor free graph with $\left|V\left(H^{\prime \prime}\right)\right|<|V(G)|$. As $u y \in E(G), c(u) \neq c(y)$. By Claim 3.1.4, we have $\max \left\{d_{G}(u), d_{G}(y)\right\} \leq r$. Hence

$$
\begin{aligned}
|c[u] \cup c[y]| & \leq d_{G}(u)+d_{G}(y)-m_{G}(y)-1 \\
& \leq d_{G}(u)+d_{G}(y)-d_{G}(u) / 2 \\
& =d_{G}(u) / 2+d_{G}(y) \\
& \leq\lfloor 3 r / 2\rfloor \\
& \leq K(r)-1 .
\end{aligned}
$$

Thus the number of colors that cannot be used for the uncolored neighbor $w^{\prime}$ of the vertices $u, y$ in $G$ is less than $K(r)$. Therefore, as $c(u) \neq c(y)$, we can extend $c$ to an $(L, r)$-coloring of $G$ by defining $c\left(w^{\prime}\right) \in L\left(w^{\prime}\right)-c[u] \cup c[y]$, contrary to (3.2).

Since in all cases, a contradiction is obtained, this establishes the theorem and completes the proof. 


\subsection{Remark}

Motivated by Wegner' conjecture and the result in this paper, it is natural to seek, for each integer $r \geq 1$, the smallest integers $f_{1}(r)$ and $f_{2}(r)$ such that for any planar graph $G, \chi_{r}(G) \leq$ $f_{1}(r)$ and $\chi_{L, r}(G) \leq f_{2}(r)$. By the Four Color Theorem $([4,5,39])$ and by the results in [8], we believe that the following holds.

Conjecture 3.2.1. Let $G$ be a planar graph. Then we have $\chi_{r}(G) \leq f_{1}(r)$, where

$$
f_{1}(r)= \begin{cases}r+3, & \text { if } 1 \leq r \leq 2 \\ r+5, & \text { if } 3 \leq r \leq 7 \\ \lfloor 3 r / 2\rfloor+1, & \text { if } r \geq 8\end{cases}
$$




\section{Chapter 4}

\section{The quantitative description of VEGF receptor localization based on hierarchical clustering}

\subsection{Methods}

\section{Experimental details}

We study TEM (transmission electron microscopy) images or PAE-KDR cells, porcine aortic endothelial cells that artificially express VEGFR-2 (KDR) receptors. The receptors are labelled with $10 \mathrm{~nm}$ diameter gold particles [50]. Here, each labelled receptor is represented by a dark spot. The locations of the spots are used to determine the location of receptors with high precision. Sources of uncertainty include the size of the probe and that of the molecule. The images should be interpreted as "snapshots" of the position of the receptors, at the moment when the cell was prepared for imaging.

Sources of position uncertainty include the size of the probe and that of the molecule. Since the diameter of the probe is approximately $10 \mathrm{~nm}$, inter-receptor separations smaller than this size can not be resolved and the two receptors are recorded as a single particle. This is a likely explanation of the exclusion distances observed on the nearest neighbor distance plots in Figures 4.6 and 4.7 .

The coordinates of the receptors are obtained by identifying the centers of the respective dark spots on the micrographs. Currently this is done using a program called ImageJ, which provides various tools to enhance and map the image files. The procedure is semi-automatic in the sense that a good fraction of the cooridnates are obtained by manually correcting the positions obtained by the program $[13,50]$. The positions are then downloaded into a text 

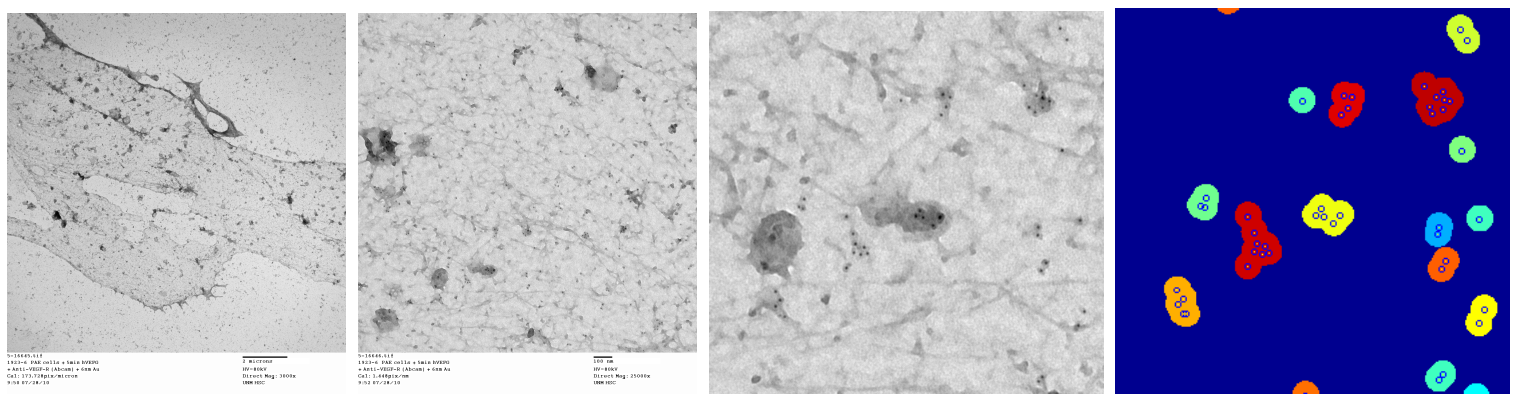

Figure 4.1: Transmission electron microscopy (TEM) images of nano-gold labelled VEGF receptors on the membrane of PAE-KDR cells. The images shown are details of the ones to their left. The gold particles appear as dark spots, whose cordinates are extracted in a semi-automatic procedure. The last image shows the clusters obtained by our program.

file. All subsequent analysis was performed through automated algorithms implemented by the authors.

\section{Traditional measures of clustering}

The spatial distribution of receptors, as seen in microscopic images, is generally not random, and apears so to a casual observer. However, a rational definition of clusters, leading to an identification similar to the one shown in Figure 4.2 is not obvious. Clustering, defined as the accumulation of receptors in a small fraction of the available area, has a likely impact on signaling, therefore quantitative measures are a crucial ingredient to predictive mathematical models of cell signaling. The traditional methods used in the field are often focused on establishing the fact of clustering, by comparison with analytically known distributions that would correspond to a random placement.

A widespread approach to cluster analysis relies on the mutual (Euclidean) distance between pairs of points. The nearest-neighbor distance (NDD) is obtained by selecting, for each point in a distribution, the point from the rest of the set that is the closest. The nearest-neighbor distance gives a measure of the local density of points. If points are distributed uniformly, then the average nearest-neighbor distance should be close to the radius of the area available to each point. The actual distribution can be derived analytically for a random configuration.

\section{Hierarchic clustering}

The method of distance based hierarchic clustering has been applied in various contexts [21] Our starting point is the work of Espinoza and coworkers [13], who initially adapted the method to the analysis of nano-gold labelled membrane proteins. 

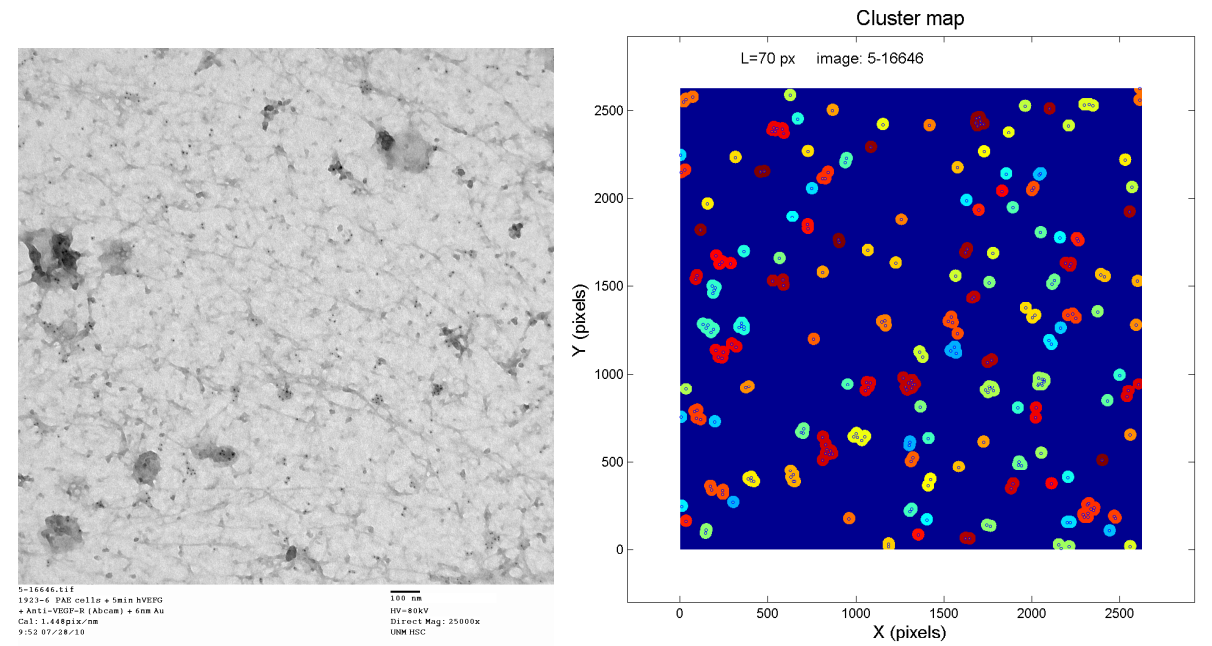

Figure 4.2: The fact that the dark spots are clustered is readily apparent to a casual observer. However, a reliable, rational definition of clusters that properly conveys the subjective notion, and can be compared with model predictions, requires careful mathematical construction. The image on the right is the result of hierarchical clustering analysis applied to the TEM image on the left.

Given a set of points $\mathcal{P}=\{\mathrm{A}, \mathrm{B}, \cdots\}$ in a space endowed with a measure of distance $d($,$) ,$ we may group the points into distance based clusters defined by a chosen scale $L \geq 0$ as follows.

i. Two points $(A, B)$ are in the same cluster if their distance is less than $L$,

$$
d(\mathrm{~A}, \mathrm{~B}) \leq L \Rightarrow \mathrm{A} \sim_{\mathrm{L}} \mathrm{B}
$$

ii. The relation $A \sim_{L} B$ is extended by transitivity, i.e., two points are in the same cluster if there is a third point which is in the same cluster with each of them:

$$
\left(A \sim_{L} B \quad \& A \sim_{L} C\right) \Rightarrow B \sim_{L} C .
$$

The relation $A \sim_{L} B$ defined above is also symmetric, and is therefore an equivalence relation on the set of points $\mathcal{P}$. Clusters are uniquely defined as the equivalence classes induced by $\sim_{\mathrm{L}}$.

\section{Cluster number versus length scale}

The scale $L$ is crucial to the definition of the clusters. If we compare the clusters induced by two different scales $L_{1}<L_{2}$, on the same set of points, we easily find that two points that are in the same cluster with respect to the shorter scale are always in the same cluster with respect to the longer scale,

$$
\left(L_{1} \leq L_{2}\right) \Rightarrow\left((\forall) \mathrm{A}, \mathrm{B} \in \mathcal{P}, \quad \mathrm{A} \sim_{\mathrm{L}_{1}} \mathrm{~B} \Rightarrow \mathrm{A} \sim_{\mathrm{L}_{2}} \mathrm{~B}\right) .
$$




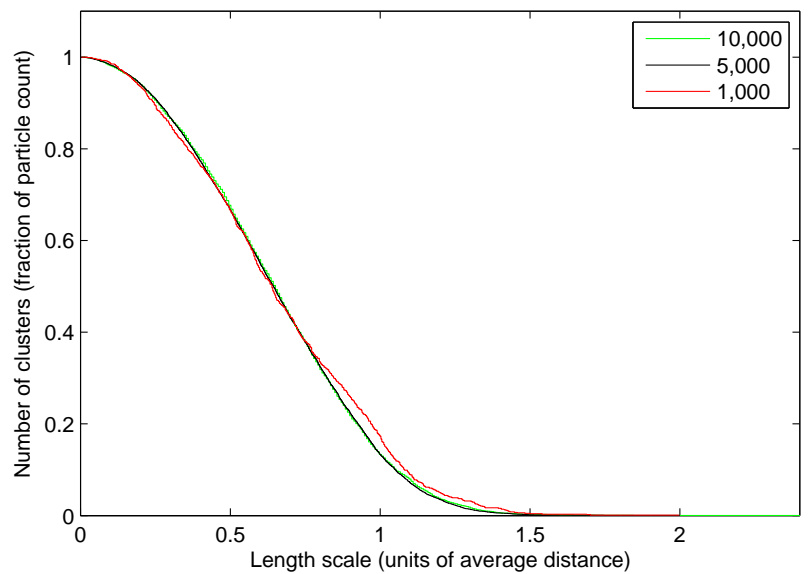

Figure 4.3: The dependence of the number of clusters $N_{\mathrm{C}}$ on the length scale $L$ (for $N_{\text {total }}$ points distributed randomly in a square of area $\left.A_{\text {total }}\right)$ appears to follow a universal function as shown in Eq. 4.4. The curves shown are from $N_{\text {total }}=\{1000,5000,10000\}$ points randomly placed in a unit square.

In other words, each cluster induced by $L_{1}$ is a subset of a cluster induced by $L_{2}$, and as the length parameter is increased, the clusters grow in size and their number decreases.

One way to characterize the distribution of particles is to analyze the dependence of the number of clusters on the lengh parameter, $N_{C}(L)$. We have established that $N_{\mathrm{C}}(L)$ is a decreasing function for any given set of points. As $L \rightarrow 0$, each point in $\mathcal{P}$ is its own cluster, and as $L$ increases beyond the largest point to point distance, the entire set $\mathcal{P}$ forms a single cluster. ${ }^{1}$

We found through numerical simulations that the curve for randomly distributed points in an area can be obtained as a scaled version of a universal function, as shown in Figure 4.3. The scaling law is as follows:

$$
N_{\mathrm{C}}(L)=N_{\text {total }} \cdot \phi\left(\frac{L}{\sqrt{A_{\text {total }} / N_{\text {total }}}}\right) .
$$

The knowledge of this universal function is useful in comparing our experimentally derived distributions with corresponding random sets of points.

\section{High affinity domains and cluster sizes}

Assuming that we have identified the clusters in a set of images, we can use the distribution of cluster sizes to test our hypotheses regarding the mechanism behind clustering. Below we outline

\footnotetext{
${ }^{1}$ The analysis presented here differs from that in [13], which focused on clusters of two or more particles. The curve obtained there would approach 0 , respectively 1 at small and large values of $L$, with at least one maximum in between.
} 
a predictive model for cluster distributions based on the hypothesis of pre-existing high affinity microdomains. These are small areas in the cell membrane that are atractive to receptors, in the sense that rceceptors spend a relatively high fraction of theri time in these regions. Such attractive areas could be, for example, lipid rafts, protein islands, or regions immediately adjacent to elements of the cytoskeleton. We do not attempt to discuss here the specific mechanism behind the attractiveness of thse domains.

We assume that the image we observe (e.g. the left picture in 4.2) is a small, representative fraction (patch) of the entire cell membrane, so that cell $=Q \cdot$ one patch with $Q \gg 1$. We also assume that the pre-existing high affinity domains (that eventually hold the receptors clusters) are of approximately equal size. We divide a cell into $B$ regions of this size. We call these regions 'boxes' as we are going to discuss the experiments of throwing particles into these boxes. Let the number of boxes in high (respectively low) receptor density domain of a patch be $B_{h}$ (respectively $B_{l}$ ). Let the total number of particles in high (respectively low) density domains of a patch be $R_{h}$ ( $R_{l}$ resp.). Denote the average number of particles per (any) box as $\lambda=\frac{R}{B}$, where $R=R_{h}+R_{l}$ and $B=B_{h}+B_{l}$.

The area of the cell (and the patch of interest) is thus divided between a high (receptor) density and a low density sector. We denote by $f$ the area fraction occupied by the high density sector. If the area of the cell is $A$, then the area of high density domains will be $A_{h}=f A$, and the rest, $A_{l}=(1-f) A$ coresponds to the low density region. In terms of actual domains or 'boxes', the number of boxes in the high (respectively low) density region is $B_{h}=f(B)$ $\left(B_{l}=(1-f)(B)\right.$ resp.). The attractiveness of the high density domains is $\alpha$. The ratio of the number of receptors in the high- and low-density domains is $\alpha$ times higher than that of the respective areas: $\frac{R_{h}}{R_{l}}=\alpha \frac{B_{h}}{B_{l}}$. Therefore, $R_{h}=R_{1+\alpha f}$ and $R_{l}=R_{1+\alpha f}$ (see [9] for further discussion).

In general, if we did not distinguish between high and low density boxes (and assuming $Q \gg k$, i.e. the membrane has much more patches than the size of a cluster), the probability that one box has exactly $k$ particles is given by a Poisson distribution, $f_{k}=e^{-\lambda} \cdot \lambda^{k} / k$ ! where $\lambda=R / B$ is the average number of particles per box. Consequently, the probability for one particle to be found in a box that has $k$ particles (including itself) is

$$
g_{k} \equiv f_{k-1}=e^{-\lambda} \cdot \frac{\lambda^{k-1}}{(k-1) !}
$$

In the two-sector case, the probability that one particle falls into the high density region is $R_{h} / R$, the prob that it falls in the low density region is $R_{l} / R$, that is

$$
p_{h} \equiv \frac{R_{h}}{R}=\frac{\alpha f}{1+\alpha \cdot f} \quad, \quad p_{l} \equiv \frac{R_{l}}{R}=\frac{1}{1+\alpha \cdot f} .
$$




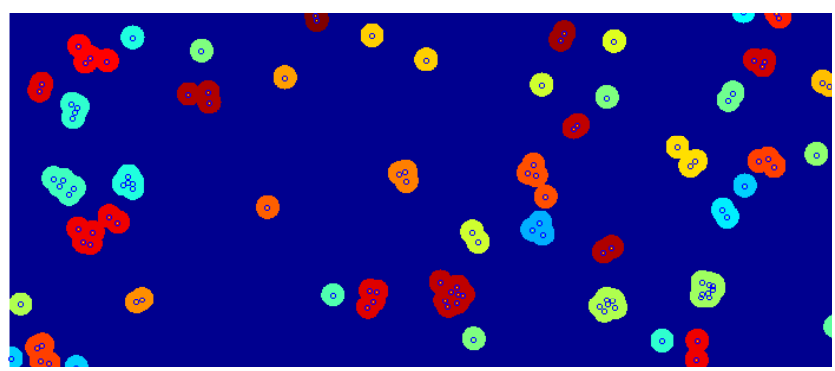

Figure 4.4: Detail of the cluster map shown in Figure 4.2. Disks of radius $L=48.9 \mathrm{~nm}$ (70 pixels) are drawn around each point. Disks corresponding to the same cluster have the same color.

Apply (4.5) to each sector, with $\lambda_{h}$ and $\lambda_{l}$ defined as

$$
\lambda_{h}=\frac{R_{h}}{B_{h}}=\frac{\alpha \lambda}{1+\alpha \cdot f} \quad, \quad \lambda_{l}=\frac{R_{l}}{B_{l}}=\frac{\lambda}{(1+\alpha \cdot f) \cdot(1-f)}
$$

to finally obtain

$$
g_{k}^{(h)}=p_{h} \cdot e^{-\lambda_{h}} \cdot \frac{\lambda_{h}^{k-1}}{(k-1) !} \quad ; \quad g_{k}^{(l)}=p_{l} \cdot e^{-\lambda_{l}} \cdot \frac{\lambda_{l}^{k-1}}{(k-1) !} .
$$

The probability that a particle is in any box with $k$ particles total, is $g_{k}=g_{k}^{(h)}+g_{k}^{(l)}$. Substituting (4.6), (4.7) and (4.8) into $g_{k}$, we have a model prediction for the distribution of particles by cluster size, in terms of the parameters $\alpha, f, R$ and $B$. For a given image or set of images the total number of particles is known ( $R=2485$ total for the 19 images discussed here). We will use this to estimate $\alpha, f$ and $B$ by a least square fit to the observed cluster size distributions.

\subsection{Results and discussion}

\section{Cluster number distributions}

We constructed clusters for values of the distance parameter ranging from $0.698 \mathrm{~nm}$ (1 pixel) to a few hundred, in order to obtain the full $N_{\mathrm{C}}(L)$ dependence. We can visualise the distribution of clusters for various length scales. A higher resolution detail of the cluster map shown in Figure 4.2 is shown in Figure 4.4. We draw a disk of radius approximately equal to the length scale (48.89 $\mathrm{nm}$ in this case) around each point. The colors are used to indicate the different clusters.

The dependence of the number of clusters on the length scale is plotted in Figure 4.5, for a single image as well as for the entire set analyzed. Comparison with the random distribution (blue lines) shows clear and consistent deviations. Both the individual image and the cumulative 

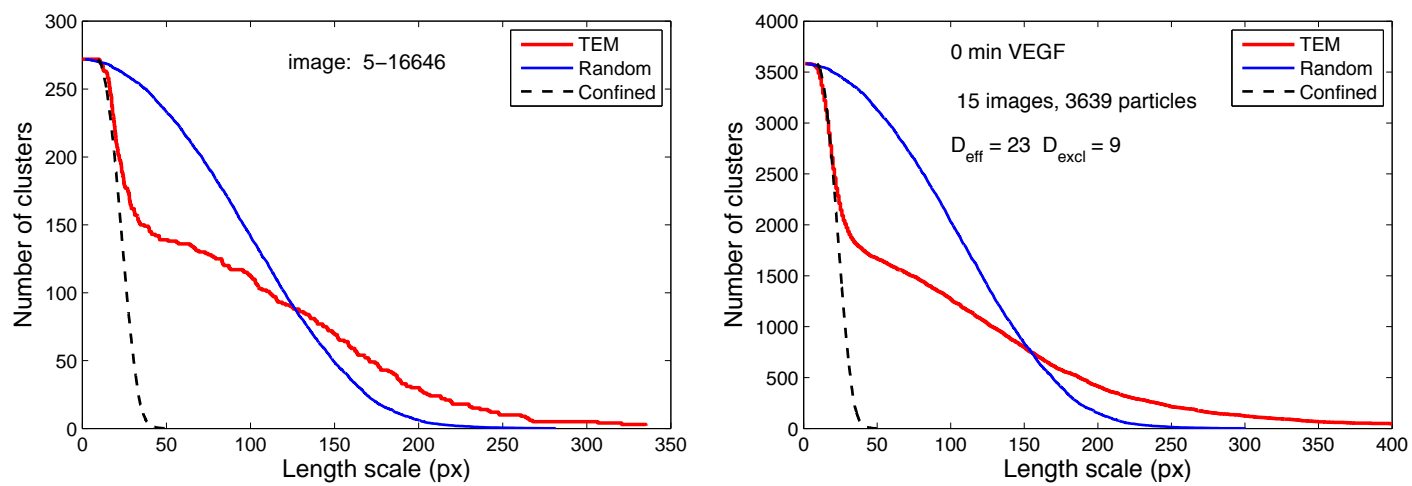

Figure 4.5: Number of clusters as a function of the length parameter (red lines) in a single TEM image (left), and a set of 15 images (right), compared to the average number of clusters expected from a random distribution with the same number points in the same area (blue lines).

plot exhibit a sharp initial decrease, followed by a significantly slower variation. The initial fast decrease corresponds to the fact that particles are much closer to their neighbors than the average distance. In an ideal clustered scenario, where the intr-cluster separation between particles is smaller than the shortest distance between clusters, the number of clusters would decrease until the largest intra-cluster distance, and one would then obseve a plateau until $L$ becomes comparable to the inter-cluster distance. While we do not observe a perfect scale separation, both the single image and the cumulative curves exhibit a clear change in behavior around 27.93 $\mathrm{nm}$ (40 pixels), and a shoulder that extends to approximately $48.89 \mathrm{~nm}$ (70 pixels).

\section{Nearest neighbor distance distributions}

We also constructed nearest-neighbor and next nearest neighbor distance distributions for all the images. Similarly to the cluster analysis results, the NND distributions (Fig. 4.6) obtained from our set of TEM imagesshow consistent deviations from the random prediction. A significant fraction of the distribution (larger in the nearest-neighbor case) is well approximated by the theoretical distribution corresponding to a smaller inter-particle distance, with a correction for a minimal separation. A smaller fraction of the distances is outside this high density mode.

The next nearest neighbor distributions (Fig. 4.7) may be more relevant to clustering, since VEGF receptors may form dimers, and thus the nearest neighbor of a receptor may be its dimer partner. They exhibit the same features as the nearest neighbor distributions; the majority of the distance distribution falls in a high density mode that is well approximated by the dashed curve. Compared to Figure 4.6, a larger fraction of the distances in Fig. 4.7 falls outside the high density mode, possibly forming a second mode that corresponds to inter-cluster distances. 

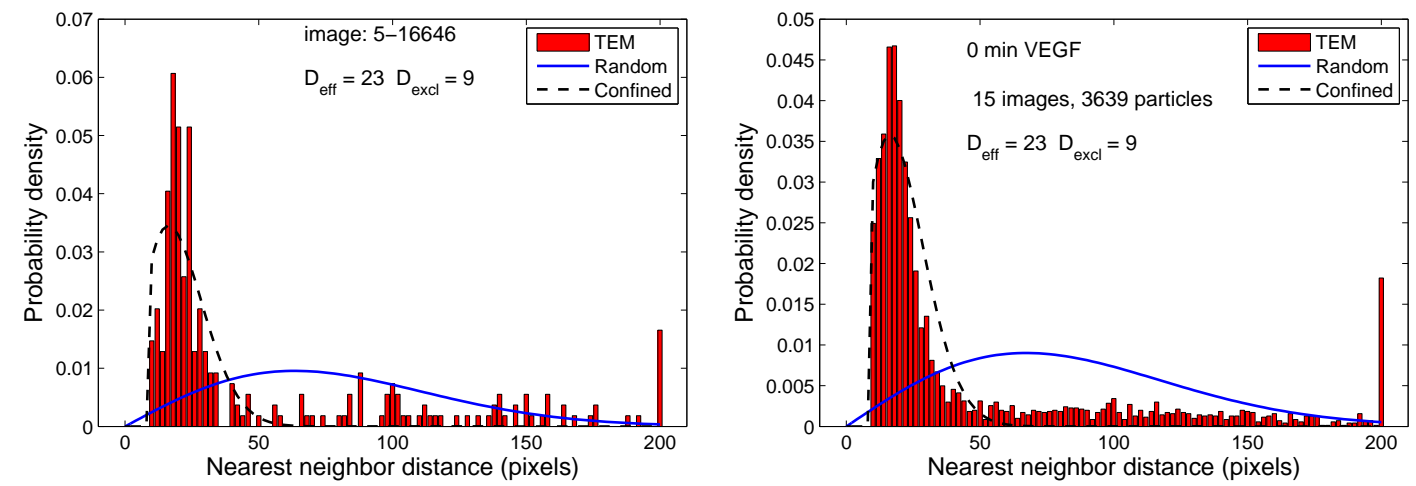

Figure 4.6: Nearest-neighbor distance distributions obtained from a single TEM image (left), and from a set of 15 similar images (right). Solid blue lines indicate the theoretical distributions corresponding to the same number of receptors distributed randomly in the same area.
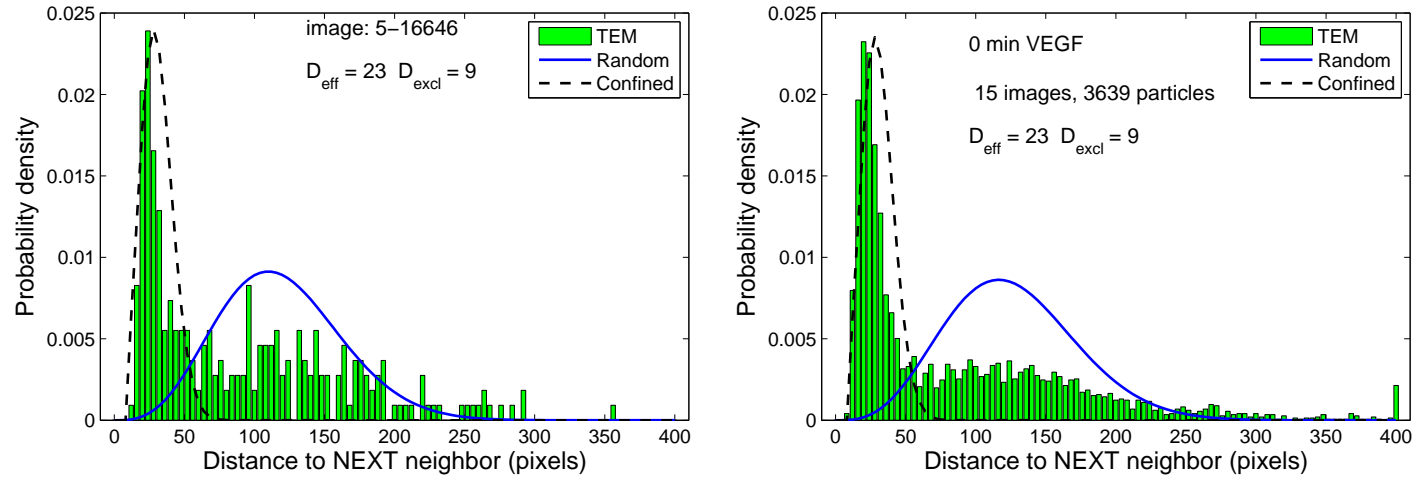

Figure 4.7: Next nearest-neighbor distance distributions corresponding to Figure 4.6 


\section{Extracting a "natural" cluster size parameter}

The comparisons we made with random distributions are compelling evidence of a behavior that is not consistent with pure random placement. The likelihood of observing our results in a random distribution are astronomically small. However, the ultimate goal of our cluster analysis is to characterize the distribution of receptors in a way that allows for quantitative comparisons between diverse cells, receptors and experimental settings, as well as a mechanistic understanding of the drivers of the observed behavior.

Similarly to previous work on hierarchical, distance based clustering, the first objective is to derive a natural length scale. If the particles are grouped into naturally defined clusters (in the sense of a clear scale separation between intra- and inter-cluster distances), this "natural" length scale can be interpreted either as the typical, or the largest intra-cluster distance between a particle and its nearest neighbor. The second definition seems more practical since this would result in correctly separating the particles into clusters. A precise mathematical definition would be the point on the $N_{\mathrm{C}}(L)$ curve with the lowest slope, which falls approximately at $48.89 \mathrm{~nm}$ (70 pixels).

\section{Toward a quantitative model of clustering}

Another approach to understanding the observed distributions is to attempt to reconstruct them based on a hypothesis regarding the mechanism of clustering. The dashed lines in Figures 4.5,4.6, and 4.7 have been obtained using a rudimentary "model", as follows. We first scaled the theoretical (random) distributions to correspond to a higher particle density $D_{\text {eff }}$. We then added a correction to take into account a minimal separation of points, by setting the corresponding

pdf. $p(x)$ to zero for $x \leq D_{\text {exclusion }}$ in the case of neareast neighbor distance distributions, and by shifting the entire cluster size distribution by the exclusion distance $D_{\text {exclusion. }}$. Finally, the curves were scaled vertically to match the integral of the high density mode (NND), respectively the total number of points (clustering).

The high density model described above only explains the first mode of the observed cluster number distributions and of the nearest neighbor distance plots. The rest of the distribution is somewhat similar to a lower density random distribution, that may reflect the inter-cluster spacing. One future line of investigaton would be to attempt to reproduce both modes, based on a model of randomly distributed clusters, consisting of particles randomly distributed at $\mathrm{q}$ high density. The typical size of a cluster could be inferred from the distribution of the number of particles in a cluster, which was not discussed here due to time constraints. 

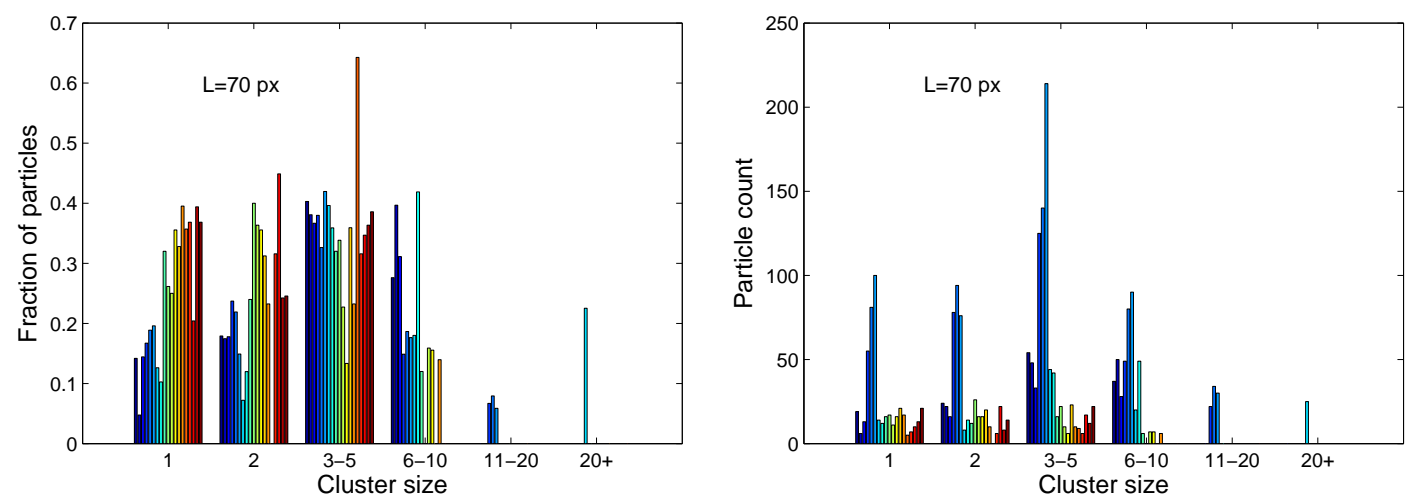

Figure 4.8: Distribution of particles by cluster size, in each of the 19 images we analyzed. Each color represents the distribution of one image. We excluded one very large cluster (a cluster contains 160 receptors) from the analysis.

\section{Parameter estimation from cluster size distributions}

We analyzed a group of 19 images of cells prepared under similar conditions. The number of observed labels varied from as low as a few tens to approximately 500. The individual distributions of particles by cluster size (grouped in intervals for larger clusters) are shown in Figure 4.8.

We compared the model predictions for the cluster size distributions $g_{k}$ with the experimental data using a least square distance between the model and experimental cluster size distributions, as shown in Figure 4.9. In the results shown, the fit was performed using a simulated annealing (Metropolis-Hastings) algorithm that quickly identified a range of good fits. The best fit shown in the figures corresponds to $B=354$ 'boxes' per image, an area fraction of $f=21 \%$ and attractiveness of $\alpha=24$ for the high affinity domains.In this optimization run, we limited $f<0.25$ and $B>200$. $B$ corresponds to the typical size of a single attractive domain and should therefore be comparable to the typical area occupied by a cluster. Fits of similar optimality can be obtained with significantly different parameter sets, for example $\alpha=4, f=31 \%$, and $B=65$ obtained without imposing the limitations mentioned above. Therefore this result should not be interpreted as a measurement of these quantitites, but rather as a proof of the principle that the observed clsuter size distributions are consistent with the high density domain hypothesis. 


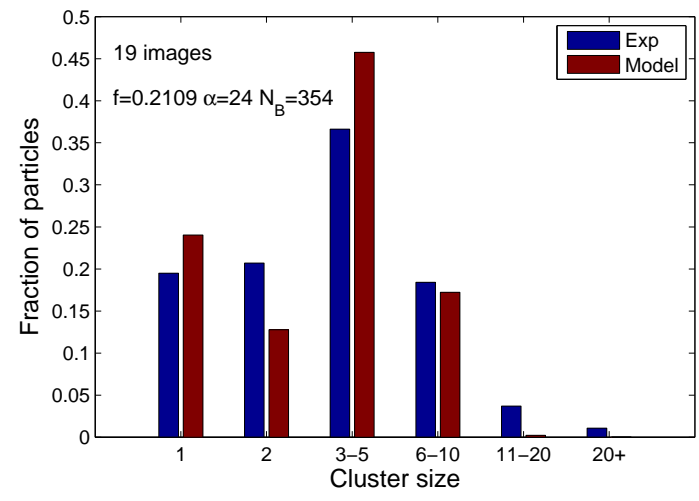

Figure 4.9: Comparison between the probability mass function $g_{k}$ with the parameters $\alpha=24$, $f=0.21, B=354$ to the experimental data. The experimental data is the aggregate of 19 images, with one very large size cluster (a cluster contains 160 receptors) omitted. 


\section{Chapter 5}

\section{Quantitative description and impact of VEGF receptor localization}

\subsection{From microscopic details to global behavior}

Our model building program relies on a sequence of models, with three different levels of detail. Abstractions and/or average behaviors obtained from one level serve as inputs to the next, higher level. We use the idea of high affinity patches as a working hypothesis.

1. At the microscopic level, we investigate the localization, motion and interactions of $i n$ dividual receptors. As discussed in previous chapter, clusters are not consistent with a random distribution. The identification of clusters can be done by a hierarchic clustering algorithm.

The observed receptor trajectories exhibit anomalous diffusion. We model this with random walks in the presence of various geometries of semi-permeable barriers. Comparisons of simulated and experimental step size distributions also support the high density patch hypothesis. In summary, the microscopic data combined with a Brownian motion model can provide estimations of the individual and combined size, as well as the attractiveness of the high density patches. In addition, direct measurements based on SPT can provide exit and entrance rates as well as dimerization and dissociation rates for molecular species of interest.

2. The information on the size and properties of high affinity patches is used at the intermediate, mesoscopic level, to simulate the reactions and interchange of receptors and receptor-ligand complexes. At this level, each high density patch is abstracted into a single, well mixed compartment. Since receptors tend to diffuse quickly through the non-attractive region until they are [re]trapped by an attractive patch, the entire non-attractive region is represented as a single compartment. The mesoscopic model is an abstraction of the microscopic models, where spatial degrees of freedom have been discretized. Mobility information is encapsulated in the particle exchange rates between domains, the capacity of the corresponding compartments, as well as 

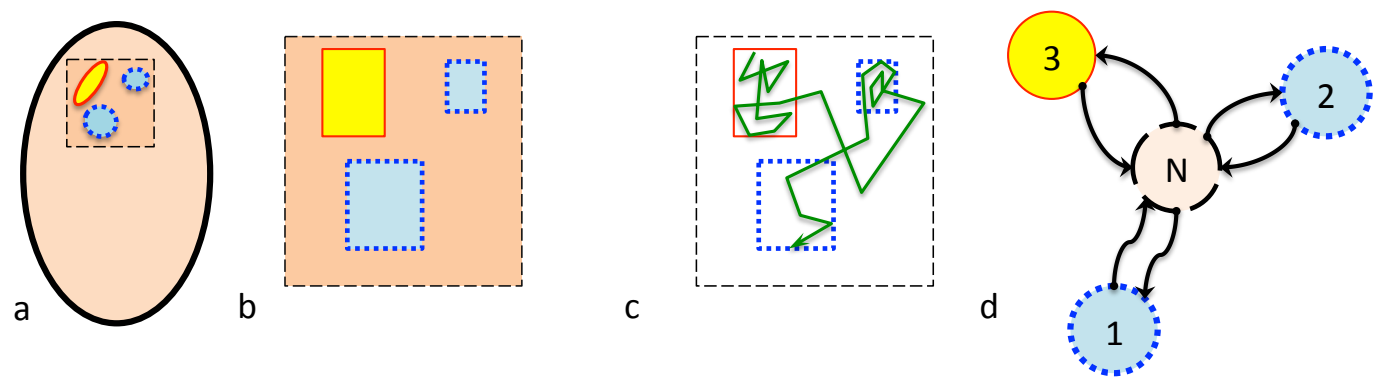

Figure 5.1: (a,b) Attractive microdomains occupy a small fraction of the cell membrane, and their measurements may be extracted from experimental images. (c) Although the receptors can move through the non-attractive regions, they tend to remain in the smaller clusters. (d) In the mesoscopic approach each microdomain, as well as the rest of the membrane (the "normal" region), are represented as well-mixed compartments that may exchange particles.

the effective dimerization rates with the compartments. In terms of implementation, this level requires the composition of a spatial network of domains, defined by an oriented, weighted adjacency graph, and a chemical reaction network.

3. The third, highest level of abstraction is obtained by (1) merging all attractive patches into a single one and (2) making the continuum approximation. The resulting ODE system is discussed in the remainder of this paper.

\subsection{ODE model and steady states}

Consistent with the emerging experimental picture, we make two assumptions. First, we assume that a fraction $f \leq 1$ of the membrane is covered by domains that have a physical affinity for receptors. As receptors diffuse throughout the membrane, the probability of crossing the boundary of such an attractive domain is asymmetric - all else being equal, inbound crossing is $\alpha \geq 1$ times more likely than outbound. We will consider the aggregate of the high affinity patches as a single high density domain, and refer to the rest as the low density domain or sector. Second, we will set the mobility of receptor dimers lower than that of monomers. These two ingredients result in the preferential accumulation of receptors in the high density patches. We investigate the effect of this accumulation (clustering) on dimerization and signal initiation. We are especially interested in establishing whether there is a postive feed-back between dimerization and receptor clustering.

\section{Reactions and Equations}

We follow the mathematical modeling framework of MacGabhann, Popel and coworkers [16] to describe free $(\mathrm{R})$ and ligand-bound (VR) receptors, receptor dimers (RR), and three ligandbound dimer complexes (VRR, RVR, $\Delta$ ); the ligand is considered constant. Their structure and 


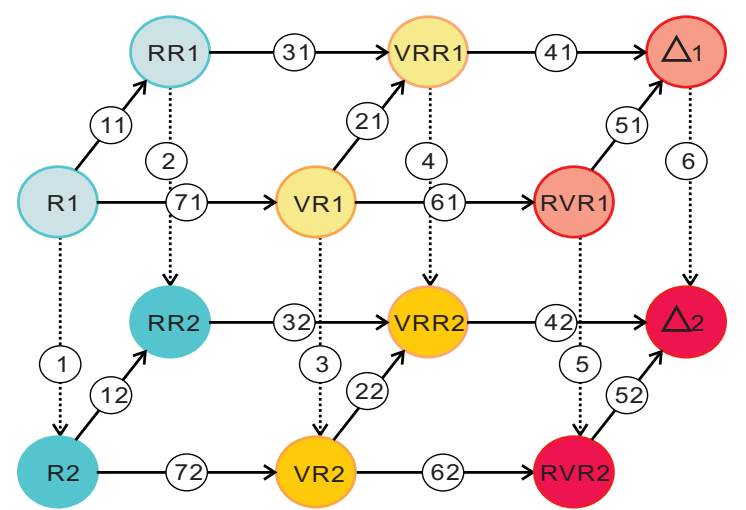

Figure 5.2: Reactions in the two-compartment model. Each horizontal sheet contains the reactions in one of the domains; transport reactions are "vertical". Here we omitted the added monomer receptors in reactions $\mathcal{C}_{1 \mathrm{x}}$ and $\mathcal{C}_{2 \mathrm{x}}$, as well as the added VEGF $(V)$ in reactions $\mathcal{C}_{3 \mathrm{x}}$ and $\mathcal{C}_{7 \mathrm{x}}$.

reactions among them are illustrated in Figure 1.1. For simplification, we assume that there is a region in the membrane with high affinity for VEGF receptors, and describe the rest as a second, "normal" or low affinity one. Each of the six species is presented in both domains; similary, each of the 7 reactions has a copy in each domain, see Figure 5.2. Assuming the free VEGF concentration is kept constant at $V_{0}$, we have a 12 -dimensional state vector,

$$
\mathbf{X}=\left(\left[\mathrm{R}_{1}\right],\left[\mathrm{R}_{2}\right],\left[\mathrm{RR}_{1}\right],\left[\mathrm{RR}_{2}\right],\left[\mathrm{VR}_{1}\right],\left[\mathrm{VR}_{2}\right],\left[\mathrm{VRR}_{1}\right],\left[\mathrm{VRR}_{2}\right],\left[\mathrm{RVR}_{1}\right],\left[\mathrm{RVR}_{2}\right],\left[\Delta_{1}\right],\left[\Delta_{2}\right]\right)^{T} .
$$

In addition to the 28 (irreversible) reactions that represent molecular transformations, we describe the transfer of every molecular species between domains as a separate reaction, bringing the total to 40 (irreversible) reactions. It is convenient to group pairs of opposing reactions into single reversible reactions [24], leaving us with 20 reversible reactions, as illustrated in Figure 5.2. The arrows represent the conventional direction for the corresponding fluxes. The 20 reactions with reaction rates assuming mass-action are denoted as follows (where $x=1,2$ ):

$$
\begin{array}{lll}
\mathcal{C}_{1 \mathrm{x}}: \mathrm{R}_{\mathrm{x}}+\mathrm{R}_{\mathrm{x}} \underset{d}{\stackrel{b}{\rightleftharpoons}} \mathrm{RR}_{\mathrm{x}} & \mathcal{C}_{2 \mathrm{x}}: \mathrm{VR}_{\mathrm{x}}+\mathrm{R}_{\mathrm{x}} \underset{d}{\stackrel{b}{\rightleftharpoons}} \mathrm{VRR}_{\mathrm{x}} ; & \mathcal{C}_{3 \mathrm{x}}: \mathrm{RR}_{\mathrm{x}}+\mathrm{V}_{0} \underset{c}{\stackrel{2 a}{\rightleftharpoons}} \mathrm{VRR}_{\mathrm{x}} ; \\
\mathcal{C}_{4 \mathrm{x}}: \mathrm{VRR}_{\mathrm{x}} \underset{c_{i}}{\stackrel{a_{i}}{\rightleftharpoons}} \Delta_{\mathrm{x}} ; & \mathcal{C}_{5 \mathrm{x}}: \mathrm{RVR}_{\mathrm{x}} \underset{d_{i}}{\stackrel{b_{i}}{\rightleftharpoons}} \Delta_{\mathrm{x}} ; & \mathcal{C}_{6 \mathrm{x}}: \mathrm{VR}_{\mathrm{x}}+\mathrm{R}_{\mathrm{x}} \stackrel{a_{s}}{\rightleftharpoons} \mathrm{RVR}_{\mathrm{x}} ; \\
& \mathcal{C}_{7 \mathrm{x}}: \mathrm{R}_{\mathrm{x}}+\mathrm{V}_{0} \underset{c}{\stackrel{a}{\rightleftharpoons}} \mathrm{VR}_{\mathrm{x}} ; & \\
\mathcal{D}_{1}: \mathrm{R}_{1} \underset{k_{2}}{\stackrel{k_{1}}{\rightleftharpoons}} \mathrm{R}_{2} ; & \mathcal{D}_{2}: \mathrm{RR}_{1} \underset{\beta k_{2}}{\stackrel{\beta k_{1}}{\rightleftharpoons}} \mathrm{RR}_{2} ; & \mathcal{D}_{3}: \mathrm{VR}_{1} \underset{k_{2}}{\stackrel{k_{1}}{\rightleftharpoons}} \mathrm{VR}_{2} ; \\
\mathcal{D}_{4}: \mathrm{VRR}_{1} \underset{\beta k_{2}}{\stackrel{\beta k_{1}}{\rightleftharpoons}} \mathrm{VRR}_{2} ; & \mathcal{D}_{5}: \mathrm{RVR}_{1} \underset{\beta k_{2}}{\stackrel{\beta k_{1}}{\rightleftharpoons}} \mathrm{RVR}_{2} ; & \mathcal{D}_{6}: \Delta_{1} \underset{\beta k_{2}}{\stackrel{\beta k_{1}}{\rightleftharpoons}} \Delta_{2} .
\end{array}
$$




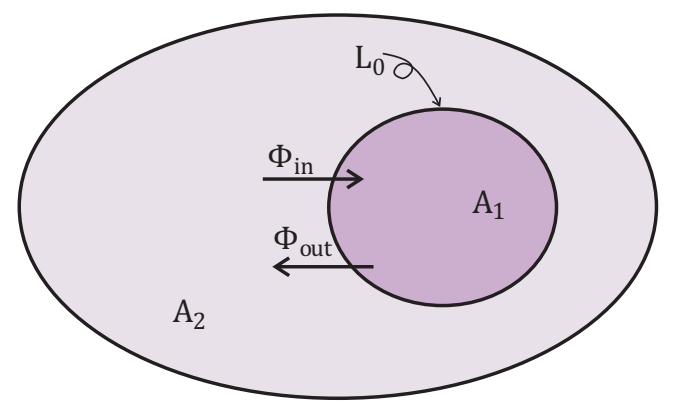

Figure 5.3: Schematic and notations for the high- and low-density areas on the cell surface. We assume that a fraction of the membrane (area $A_{1}$ ) has a higher affinity for VEGF receptors than the rest of the membrane. This translates into asymmetric rate 'constants' for the $\Phi_{\text {in }}$, and $\Phi_{\text {out }}$ fluxes.

The corresponding stoichiometry matrix is

$$
\Gamma=\left[\begin{array}{cccccccccccccccccccc}
-2 & 0 & -1 & 0 & 0 & 0 & 0 & 0 & 0 & 0 & -1 & 0 & -1 & 0 & -1 & 0 & 0 & 0 & 0 & 0 \\
0 & -2 & 0 & -1 & 0 & 0 & 0 & 0 & 0 & 0 & 0 & -1 & 0 & -1 & 1 & 0 & 0 & 0 & 0 & 0 \\
1 & 0 & 0 & 0 & -1 & 0 & 0 & 0 & 0 & 0 & 0 & 0 & 0 & 0 & 0 & -1 & 0 & 0 & 0 & 0 \\
0 & 1 & 0 & 0 & 0 & -1 & 0 & 0 & 0 & 0 & 0 & 0 & 0 & 0 & 0 & 1 & 0 & 0 & 0 & 0 \\
0 & 0 & -1 & 0 & 0 & 0 & 0 & 0 & 0 & 0 & -1 & 0 & 1 & 0 & 0 & 0 & -1 & 0 & 0 & 0 \\
0 & 0 & 0 & -1 & 0 & 0 & 0 & 0 & 0 & 0 & 0 & -1 & 0 & 1 & 0 & 0 & 1 & 0 & 0 & 0 \\
0 & 0 & 1 & 0 & 1 & 0 & -1 & 0 & 0 & 0 & 0 & 0 & 0 & 0 & 0 & 0 & 0 & -1 & 0 & 0 \\
0 & 0 & 0 & 1 & 0 & 1 & 0 & -1 & 0 & 0 & 0 & 0 & 0 & 0 & 0 & 0 & 0 & 1 & 0 & 0 \\
0 & 0 & 0 & 0 & 0 & 0 & 0 & 0 & -1 & 0 & 1 & 0 & 0 & 0 & 0 & 0 & 0 & 0 & -1 & 0 \\
0 & 0 & 0 & 0 & 0 & 0 & 0 & 0 & 0 & -1 & 0 & 1 & 0 & 0 & 0 & 0 & 0 & 0 & 1 & 0 \\
0 & 0 & 0 & 0 & 0 & 0 & 1 & 0 & 1 & 0 & 0 & 0 & 0 & 0 & 0 & 0 & 0 & 0 & 0 & -1 \\
0 & 0 & 0 & 0 & 0 & 0 & 0 & 1 & 0 & 1 & 0 & 0 & 0 & 0 & 0 & 0 & 0 & 0 & 0 & 1
\end{array}\right] .
$$

Effective concentrations: We will use effective concentrations to describe the amounts of each species found in the two domains; $\left[\mathrm{S}_{\mathrm{x}}\right]^{\text {eff }}$ is defined as the ratio of the amount (number of mols) of substance $S$ in domain $x(x=1,2)$, divided by the total area of the cell membrane $A_{\text {cell }}$. We will refer to the usual concentrations as physical, $\left[\mathrm{S}_{\mathrm{x}}\right]^{\text {phys }}$. Generally, the meaning of the concentrations and rate constants is similar to the standard approach in [16], with some important differences as discussed below.

Consider first the exchange reactions $\left(\mathcal{D}_{1} \ldots \mathcal{D}_{6}\right.$ in eq. $\left.(5.2)\right)$, exemplified by reaction $\mathcal{D}_{1}$ : $\mathrm{R}_{1} \rightleftharpoons \mathrm{R}_{2}$. Let the fraction of the area that has high affinity to VEGF receptors be $f$. The size of the high (VEGF) density area is $A_{1}=f \cdot A_{\text {cell }}$, and the remaining area is $A_{2}=(1-f) \cdot A_{\text {cell }}$ (see Figure 5.3). Let us derive the flux of unbound receptors $\mathrm{R}$ between $A_{1}$ and $A_{2}$, represented by the reaction $\mathrm{R}_{1} \rightleftharpoons \mathrm{R}_{2}$. Let $\left[\mathrm{R}_{1}\right]^{\text {phys }}$ and $\left[\mathrm{R}_{2}\right]^{\text {phys }}$ be the physical concentrations of $\mathrm{R}$ in $A_{1}$ and $A_{2}$, respectively, defined as the amount (in fmol) of $\mathrm{R}$ in $A_{1}$ (respectively $A_{2}$ ), divided by the area $A_{1}$ (resp. $A_{2}$ ). The effective concentrations, denoted as $\left[\mathrm{R}_{1}\right]^{\text {eff }}$ ([ $\left.\mathrm{R}_{2}\right]^{\text {eff }}$ resp.), are the 
amounts of $\mathrm{R}$ in $A_{1}$ ( $A_{2}$ resp.), but divided by the total area $A_{\text {cell }}$. Therefore,

$$
\left[\mathrm{R}_{1}\right]^{\text {phys }}=\frac{\left[\mathrm{R}_{1}\right]^{\text {eff }}}{f} \text { and }\left[\mathrm{R}_{2}\right]^{\text {phys }}=\frac{\left[\mathrm{R}_{2}\right]^{\text {eff }}}{(1-f)},
$$

with units of $\mathrm{fmol} / \mathrm{cm}^{2}$ for all concentrations.

We assume that the flux of receptors, $\Phi_{\text {out }}$ (amount of substance per unit time and boundary length, in $\mathrm{fmol} /(\mathrm{cm} \cdot \mathrm{s})$ in our case), from $A_{1}$ to $A_{2}$ is proportional to the physical concentration, $\Phi_{\text {out }}=\gamma_{\text {out }}\left[\mathrm{R}_{1}\right]^{\text {phys }}$; similarly, the receptor flux into $A_{1}$ is $\Phi_{\text {in }}=\gamma_{\text {in }}\left[R_{2}\right]^{\text {phys }}$. The factors $\gamma_{\text {in }}$ and $\gamma_{\text {out }}$ reflect the physical permeability of the boundary and have units of $\mathrm{cm} / \mathrm{s}$. We define the attractiveness, $\alpha \equiv \gamma_{\text {in }} / \gamma_{\text {out }}$, to reflect the asymmetry of the permeabilities; so we have $\Phi_{\text {in }}=\gamma_{\text {in }}\left[R_{2}\right]^{\text {phys }}=\alpha \gamma_{\text {out }}\left[R_{2}\right]^{\text {phys }}$, and $\alpha \geq 1$ means that a receptor or a dimer is more easily transferred into the high concentration area $A_{1}$ than into $A_{2}$. Consequently, the exchange fluxes between the two domains will balance when the ratio of the respective physical concentrations is $\alpha$, i.e. $\Phi_{\text {in }}=\Phi_{\text {out }} \Leftrightarrow \alpha \gamma_{\text {out }}\left[\mathrm{R}_{2}\right]^{\text {phys }}=\gamma_{\text {out }}\left[\mathrm{R}_{1}\right]^{\text {phys }} \Leftrightarrow \alpha\left[\mathrm{R}_{2}\right]^{\text {phys }}=\left[\mathrm{R}_{1}\right]^{\text {phys }}$.

Consider the net rate of change of concentrations $\left[\mathrm{R}_{1}\right]^{\text {phys }}$ and $\left[\mathrm{R}_{2}\right]^{\text {phys }}$, due to the exchange of receptor monomers between the two compartments, we have

$$
\left(\frac{d\left[\mathrm{R}_{1}\right]^{\mathrm{phys}}}{d t}\right)_{\Phi}=\frac{\left(\Phi_{\mathrm{in}}-\Phi_{\mathrm{out}}\right) \cdot L_{0}}{A_{1}} ; \quad\left(\frac{d\left[\mathrm{R}_{2}\right]^{\mathrm{phys}}}{d t}\right)_{\Phi}=\frac{\left(\Phi_{\mathrm{out}}-\Phi_{\mathrm{in}}\right) \cdot L_{0}}{A_{2}}
$$

where $L_{0}$ is the length of the boundary between $A_{1}$ and $A_{2}$. Substitute $A_{1}, A_{2}, \Phi_{1}$ and $\Phi_{2}$ into $(5.4)$ :

$$
\begin{aligned}
& \left(\frac{d\left[\mathrm{R}_{1}\right]^{\text {phys }}}{d t}\right)_{\Phi}=\left(\alpha\left[\mathrm{R}_{2}\right]^{\text {phys }}-\left[\mathrm{R}_{1}\right]^{\text {phys }}\right) \frac{L_{0} \gamma_{\text {out }}}{f A_{\text {cell }}} \\
& \left(\frac{d\left[\mathrm{R}_{2}\right]^{\text {phys }}}{d t}\right)_{\Phi}=\left(\left[\mathrm{R}_{1}\right]^{\text {phys }}-\alpha\left[\mathrm{R}_{2}\right]^{\text {phys }}\right) \frac{L_{0} \gamma_{\text {out }}}{(1-f) A_{\text {cell }}} .
\end{aligned}
$$

Defining a common time constant $\delta \equiv A_{\text {cell }} /\left(L_{0} \gamma_{\text {out }}\right)$, we have

$$
\begin{aligned}
\left(\frac{d\left[\mathrm{R}_{1}\right]^{\text {phys }}}{d t}\right)_{\Phi} & =\left(\alpha\left[\mathrm{R}_{2}\right]^{\text {phys }}-\left[\mathrm{R}_{1}\right]^{\text {phys }}\right) \frac{1}{f \delta} \\
\left(\frac{d\left[\mathrm{R}_{2}\right]^{\text {phys }}}{d t}\right)_{\Phi} & =\left(\left[\mathrm{R}_{1}\right]^{\text {phys }}-\alpha\left[\mathrm{R}_{2}\right]^{\text {phys }}\right) \frac{1}{(1-f) \delta} .
\end{aligned}
$$

Finally, substituting the effective concentrations from (5.3) yields

$$
\begin{aligned}
& \left(\frac{d\left[\mathrm{R}_{1}\right]^{\mathrm{eff}}}{d t}\right)_{\Phi}=\frac{\alpha}{\delta(1-f)}\left[\mathrm{R}_{2}\right]^{\mathrm{eff}}-\frac{1}{\delta f}\left[\mathrm{R}_{1}\right]^{\mathrm{eff}}, \\
& \left(\frac{d\left[\mathrm{R}_{2}\right]^{\mathrm{eff}}}{d t}\right)_{\Phi}=-\frac{\alpha}{\delta(1-f)}\left[\mathrm{R}_{2}\right]^{\mathrm{eff}}+\frac{1}{\delta f}\left[\mathrm{R}_{1}\right]^{\mathrm{eff}} .
\end{aligned}
$$

The above result implies the identity $\frac{d\left[\mathrm{R}_{1}\right]^{\mathrm{eff}}}{d t}+\frac{d\left[\mathrm{R}_{2}\right]^{\mathrm{eff}}}{d t}=0$, which reflects particle number conservation. This is the main advantage of using effective concentrations. 
We follow the same line of reasoning for the other transfer reactions. The exit rate constants also reflect the generic mobility of particles; in a more detailed simulation, one could relate them to the diffusion constants and the permeability of the membranes. Here we will assume that exit rate constants are the same as above for ligand-bound monomer species $V R_{x}$. For dimer species, $R R_{x}, V R R_{x}, R V R_{x}$ and $\Delta_{x}$, we will use reduced exit rate constants, proportional to $\gamma_{\text {in }}$ and $\gamma_{\text {out }}$, and denote the coefficient as $\beta$. In summary, the six exchange fluxes are

$$
\begin{array}{ll}
\phi_{1}=k_{1}\left[\mathrm{R}_{1}\right]^{\mathrm{eff}}-k_{2}\left[\mathrm{R}_{2}\right]^{\mathrm{eff}}, & \phi_{2}=\beta\left(k_{1}\left[\mathrm{RR}_{1}\right]^{\mathrm{eff}}-k_{2}\left[\mathrm{RR}_{2}\right]^{\mathrm{eff}}\right), \\
\phi_{3}=k_{1}\left[\mathrm{VR}_{1}\right]^{\mathrm{eff}}-k_{2}\left[\mathrm{VR}_{2}\right]^{\mathrm{eff}}, & \phi_{4}=\beta\left(k_{1}\left[\mathrm{VRR}_{1}\right]^{\mathrm{eff}}-k_{2}\left[\mathrm{VRR}_{2}\right]^{\mathrm{eff}}\right), \\
\phi_{5}=\beta\left(k_{1}\left[\mathrm{RVR}_{1}\right]^{\mathrm{eff}}-k_{2}\left[\mathrm{RVR}_{2}\right]^{\mathrm{eff}}\right), & \phi_{6}=\beta\left(k_{1}\left[\Delta_{1}\right]^{\mathrm{eff}}-k_{2}\left[\Delta_{2}\right]^{\mathrm{eff}}\right),
\end{array}
$$

where $k_{1}=\frac{1}{\delta f}$ and $k_{2}=\frac{\alpha}{\delta(1-f)}$.

Next, we consider the rates of chemical reactions, molecular transformations that take place within each area. As an example, consider a reaction in the high density area. For $\mathcal{C}_{21}$ : $\mathrm{VR}_{1}+\mathrm{R}_{1} \underset{d}{\stackrel{b}{\rightleftharpoons}} \mathrm{VRR}_{1}$, we have

$$
\begin{aligned}
\frac{d\left[\mathrm{R}_{1}\right]^{\text {phys }}}{d t}=\frac{\left[\mathrm{dVR}_{1}\right]^{\text {phys }}}{d t}=-b\left[\mathrm{R}_{1}\right]^{\text {phys }}\left[\mathrm{VR}_{1}\right]^{\text {phys }}+d\left[\mathrm{VRR}_{1}\right]^{\text {phys }}, \\
\frac{d\left[\mathrm{VRR}_{1}\right]^{\text {phys }}}{d t}=b\left[\mathrm{R}_{1}\right]^{\text {phys }}\left[\mathrm{VR}_{1}\right]^{\text {phys }}-d\left[\mathrm{VRR}_{1}\right]^{\text {phys }} .
\end{aligned}
$$

Substituting $\left[\mathrm{R}_{1}\right]^{\mathrm{phys}}=\left[\mathrm{R}_{1}\right]^{\mathrm{eff}} / f,\left[\mathrm{VR}_{1}\right]^{\text {phys }}=\left[\mathrm{VR}_{1}\right]^{\mathrm{eff}} / f$ and $\left[\mathrm{RVR}_{1}\right]^{\mathrm{phys}}=\left[\mathrm{RVR}_{1}\right]^{\mathrm{eff}} / f$, we have

$$
\frac{d\left[\mathrm{VRR}_{1}\right]^{\mathrm{eff}}}{d t}=-\frac{d\left[\mathrm{R}_{1}\right]^{\mathrm{eff}}}{d t}=-\frac{d\left[\mathrm{VR}_{1}\right]^{\mathrm{eff}}}{d t}=\frac{b}{f}\left[\mathrm{R}_{1}\right]^{\mathrm{eff}}\left[\mathrm{VR}_{1}\right]^{\mathrm{eff}}-d\left[\mathrm{VRR}_{1}\right]^{\mathrm{eff}}
$$

therefore the flux for reaction $r_{21}$ is

$$
\phi_{21}=\frac{b}{f}\left[\mathrm{R}_{1}\right]^{\mathrm{eff}}\left[\mathrm{VR}_{1}\right]^{\mathrm{eff}}-d\left[\mathrm{VRR}_{1}\right]^{\mathrm{eff}} .
$$

The only difference between the above rate law and the one in terms of physical concentrations (5.5), is that the dimerization rate constant is scaled by the relative size of the domain, $b \rightarrow b / f$. This reflects the effect of clustering on dimerization; if the same number of reacting molecules are forced into a smaller space, their collision rate and implicitly, the absolute dimerization rate, will increase. Similar considerations give the following for the 14 reversible reactions:

$$
\begin{array}{llll}
\phi_{11} & =\frac{2 b}{f}\left[\mathrm{R}_{1}\right]^{\mathrm{eff}}{ }^{2}-d_{1}\left[\mathrm{RR}_{1}\right]^{\mathrm{eff}}, & \phi_{12} & =\frac{2 b}{1-f}\left[\mathrm{R}_{2}\right]^{\mathrm{eff}}{ }^{2}-d\left[\mathrm{RR}_{2}\right]^{\mathrm{eff}} \\
\phi_{21} & =\frac{b}{f}\left[\mathrm{R}_{1}\right]^{\mathrm{eff}}\left[\mathrm{VR}_{1}\right]^{\mathrm{eff}}-d\left[\mathrm{VRR}_{1}\right]^{\mathrm{eff}}, & \phi_{22} & =\frac{b}{1-f}\left[\mathrm{R}_{2}\right]^{\mathrm{eff}}\left[\mathrm{VR}_{2}\right]^{\mathrm{eff}}-d_{2}\left[\mathrm{VRR}_{2}\right]^{\mathrm{eff}} \\
\phi_{31} & =2 a V_{0}\left[\mathrm{RR}_{1}\right]^{\mathrm{eff}}-c\left[\mathrm{VRR}_{1}\right]^{\mathrm{eff}}, & \phi_{32} & =2 a V_{0}\left[\mathrm{RR}_{2}\right]^{\mathrm{eff}}-c\left[\mathrm{VRR}_{2}\right]^{\mathrm{eff}} \\
\phi_{41} & =a_{i}\left[\mathrm{VRR}_{1}\right]^{\mathrm{eff}}-2 c_{i}\left[\Delta_{1}\right]^{\mathrm{eff}}, & \phi_{42} & =a_{i}\left[\mathrm{VRR}_{2}\right]^{\mathrm{eff}}-2 c_{i}\left[\Delta_{2}\right]^{\mathrm{eff}} \\
\phi_{51} & =b_{i}\left[\mathrm{RVR}_{1}\right]^{\mathrm{eff}}-d_{i}\left[\Delta_{1}\right]^{\mathrm{eff}}, & \phi_{52} & =b_{i}\left[\mathrm{RVR}_{2}\right]^{\mathrm{eff}}-d_{i}\left[\Delta_{2}\right]^{\mathrm{eff}} \\
\phi_{61} & =\frac{a_{s}}{f}\left[\mathrm{R}_{1}\right]^{\mathrm{eff}}\left[\mathrm{VR}_{1}\right]^{\mathrm{eff}}-c\left[\mathrm{RVR}_{1}\right]^{\mathrm{eff}}, & \phi_{62}=\frac{a_{s}}{1-f}\left[\mathrm{R}_{2}\right]^{\mathrm{eff}}\left[\mathrm{VR}_{2}\right]^{\mathrm{eff}}-c\left[\mathrm{RVR}_{2}\right]^{\mathrm{eff}} \\
\phi_{71}=a V_{0}\left[\mathrm{R}_{1}\right]^{\mathrm{eff}}-c\left[\mathrm{VR}_{1}\right]^{\mathrm{eff}}, & \phi_{72}=a V_{0}\left[\mathrm{R}_{2}\right]^{\mathrm{eff}}-c\left[\mathrm{VR}_{2}\right]^{\mathrm{eff}} .
\end{array}
$$


We denote by $\Phi(X)=\left(\phi_{11}, \phi_{12}, \phi_{21}, \cdots, \phi_{71}, \phi_{72}, \phi_{1}, \cdots, \phi_{6}\right)^{T}$, then the system of differential equation assuming mass-action is

$$
\frac{d \mathbf{X}}{d t}=\Gamma \cdot \Phi(\mathbf{X})
$$

\section{Parameter values}

Receptor Diffusivity and Boundaries: For the diffusivity of VEGFR, we used the exit rate of $\gamma_{\text {out }}=8.23 \cdot 10^{-6} \mathrm{~cm} / \mathrm{s}$ based on the expression given in [16]. The cells are assumed to have a surface area of $1000 \mu \mathrm{m}^{2}$ [16]; assuming a spherical shape, the radius of a cell works out to approximately $r_{\text {cell }}=8.9 \mu \mathrm{m}$, and the length of the high density area boundary is

$L_{0}=2 \pi \sqrt{r_{\text {cell }}^{2}-\left(r_{\text {cell }}-\frac{1000 f}{2 \pi \cdot r_{\text {cell }}}\right)^{2}} \mu \mathrm{m}$, where $f \equiv A_{1} / A_{\text {cell }}$ is the relative fraction of the HD area. The $L_{0}$ and $\gamma_{\text {out }}$ can be readily substituted into the definition $\delta=A_{\text {cell }} /\left(L_{0} \gamma_{\text {out }}\right)$. The graph of $\delta$ as a function of $f$ is shown in Figure 5.4. We can see that $\delta$ decreases faster in the beginning as $f$ is increasing from 0 to 0.5 .

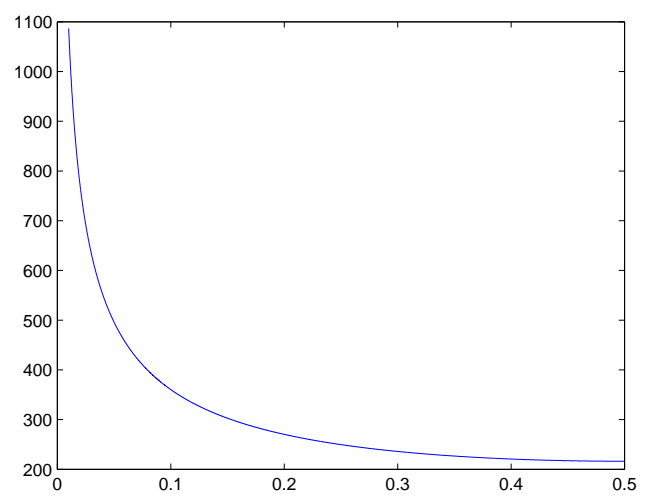

Figure 5.4: Graph of $\delta$ as a function in $f(0 \leq f \leq 0.5)$.

Following [16], we assume that there are 40,000 receptor monomers on one cell membrane, corresponding to $R_{\text {total }} \approx 6.6 \mathrm{fmol} \cdot \mathrm{cm}^{-2}$.

Reaction Rates: We use the model of [16], with base units as follows: volume concentration (of VEGF only, $V_{0}$ ), in $\mathrm{nM}$; all surface concentrations in $\mathrm{fmol} / \mathrm{cm}^{2}$; time in s. Except for VEGF, whose concentration is not a variable, all molecular species in the model are surface bound. Consequently, the units of mass-action rate constants are $\mathrm{s}^{-1}$ for unimolecular reactions, $(\mathrm{nM} \cdot \mathrm{s})^{-1}$ for reactions involving VEGF, and $\mathrm{cm}^{2} /(\mathrm{fmol} \cdot \mathrm{s})$ for on-surface dimerizations. The rate constants are summarized in the table below. 


\begin{tabular}{|c|c|c|c|c|c|c|c|c|c|}
\hline Reaction & Direction & Notation & Value & Unit & Reaction & Direction & Notation & Value & Unit \\
\hline \multirow[t]{2}{*}{$\mathrm{R}_{\mathrm{x}}+\mathrm{R}_{\mathrm{x}} \rightleftharpoons \mathrm{RR}_{\mathrm{x}}$} & $\rightarrow$ & $b$ & 0.1 & $\mathrm{~cm}^{2} /(\mathrm{fmol} \cdot \mathrm{s})$ & \multirow{2}{*}{$\mathrm{R}_{1} \rightleftharpoons \mathrm{R}_{2}$} & $\rightarrow$ & $k_{1}$ & 0.0277 & $\mathrm{~s}^{-1}$ \\
\hline & $\leftarrow$ & $d$ & 0.01 & $\mathrm{~s}^{-1}$ & & $\leftarrow$ & $k_{2}$ & 0.0154 & $\mathrm{~s}^{-1}$ \\
\hline \multirow[t]{2}{*}{$\mathrm{VR}_{\mathrm{x}}+\mathrm{R}_{\mathrm{x}} \rightleftharpoons \mathrm{VRR}_{\mathrm{x}}$} & $\rightarrow$ & $b$ & 0.1 & $\mathrm{~cm}^{2} /(\mathrm{fmol} \cdot \mathrm{s})$ & \multirow[t]{2}{*}{$\mathrm{RR}_{1} \rightleftharpoons \mathrm{RR}_{2}$} & $\rightarrow$ & $\beta k_{1}$ & 0.01385 & $\mathrm{~s}^{-1}$ \\
\hline & $\leftarrow$ & $d$ & 0.01 & $\mathrm{~s}^{-1}$ & & $\leftarrow$ & $\beta k_{2}$ & 0.0077 & $\mathrm{~s}^{-1}$ \\
\hline \multirow[t]{2}{*}{$\mathrm{RR}_{\mathrm{x}}+\mathrm{V}_{0} \rightleftharpoons \mathrm{VRR}_{\mathrm{x}}$} & $\rightarrow$ & $2 a$ & 0.0044 & $(\mathrm{nM} \cdot \mathrm{s})^{-1}$ & \multirow[t]{2}{*}{$\mathrm{VR}_{1} \rightleftharpoons \mathrm{VR}_{2}$} & $\rightarrow$ & $k_{1}$ & 0.0277 & $\mathrm{~s}^{-1}$ \\
\hline & $\leftarrow$ & $c$ & 0.026 & $\mathrm{~s}^{-1}$ & & $\leftarrow$ & $k_{2}$ & 0.0154 & $\mathrm{~s}^{-1}$ \\
\hline \multirow[t]{2}{*}{$\mathrm{VRR}_{\mathrm{x}} \rightleftharpoons \Delta_{\mathrm{x}}$} & $\rightarrow$ & $a_{i}$ & 0.949 & $\mathrm{~s}^{-1}$ & \multirow[t]{2}{*}{$\mathrm{VRR}_{1} \rightleftharpoons \mathrm{VRR}_{2}$} & $\rightarrow$ & $\beta k_{1}$ & 0.01385 & $\mathrm{~s}^{-1}$ \\
\hline & $\leftarrow$ & $c_{i}$ & 0.026 & $\mathrm{~s}^{-1}$ & & $\leftarrow$ & $\beta k_{2}$ & 0.0077 & $\mathrm{~s}^{-1}$ \\
\hline \multirow[t]{2}{*}{$\mathrm{RVR}_{\mathrm{x}} \rightleftharpoons \Delta_{\mathrm{x}}$} & $\rightarrow$ & $b_{i}$ & 0.446 & $\mathrm{~s}^{-1}$ & \multirow[t]{2}{*}{$\mathrm{RVR}_{1} \rightleftharpoons \mathrm{RVR}_{2}$} & $\rightarrow$ & $\beta k_{1}$ & 0.01385 & $\mathrm{~s}^{-1}$ \\
\hline & $\leftarrow$ & $d_{i}$ & 0.02 & $\mathrm{~s}^{-1}$ & & $\leftarrow$ & $\beta k_{2}$ & 0.0077 & $\mathrm{~s}^{-1}$ \\
\hline \multirow[t]{2}{*}{$\mathrm{VR}_{\mathrm{x}}+\mathrm{R}_{\mathrm{x}} \rightleftharpoons \mathrm{RVR}_{\mathrm{x}}$} & $\rightarrow$ & $a_{s}$ & 0.21 & $\mathrm{~cm}^{2} /(\mathrm{fmol} \cdot \mathrm{s})$ & \multirow[t]{2}{*}{$\Delta_{1} \rightleftharpoons \Delta_{2}$} & $\rightarrow$ & $\beta k_{1}$ & 0.01385 & $\mathrm{~s}^{-1}$ \\
\hline & $\leftarrow$ & $c$ & 0.026 & $\mathrm{~s}^{-1}$ & & $\leftarrow$ & $\beta k_{2}$ & 0.0077 & $\mathrm{~s}^{-1}$ \\
\hline \multirow[t]{2}{*}{$\mathrm{R}_{\mathrm{x}}+\mathrm{V}_{0} \rightleftharpoons \mathrm{VR}_{\mathrm{x}}$} & $\rightarrow$ & $a$ & 0.0044 & $(\mathrm{nM} \cdot \mathrm{s})^{-1}$ & \multirow{2}{*}{\multicolumn{5}{|c|}{$\begin{array}{l}\text { NOTE: the values for } k_{1} \text { and } k_{2} \text { given here correspond to } \\
f=0.1, \alpha=5, \beta=0.5 \text {. }\end{array}$}} \\
\hline & $\leftarrow$ & $c$ & 0.026 & $\mathrm{~s}^{-1}$ & & & & & \\
\hline
\end{tabular}

\section{Steady States}

To solve for the closed form steady-state of the differential equation system (5.8), we use the method introduced in [18]. The steady states of this system are sets of concentration values $\left\{\left[\mathrm{R}_{1}\right],\left[\mathrm{R}_{2}\right],\left[\mathrm{RR}_{1}\right],\left[\mathrm{RR}_{2}\right],\left[\mathrm{VR}_{1}\right],\left[\mathrm{VR}_{2}\right],\left[\mathrm{VRR}_{1}\right],\left[\mathrm{VRR}_{2}\right],\left[\mathrm{RVR}_{1}\right],\left[\mathrm{RVR}_{2}\right],\left[\Delta_{1}\right],\left[\Delta_{2}\right]\right\}$ for which the expression on the right-hand side of (5.8) is identically zero. Define an expanded vector $\mathbf{X}_{E}$ that consists of the original variables of $\mathbf{X}$ plus the binomials $\left[\mathrm{R}_{1}\right]^{2},\left[\mathrm{R}_{2}\right]^{2},\left[\mathrm{R}_{1}\right] \cdot\left[\mathrm{VR}_{1}\right]$ and $\left[\mathrm{R}_{2}\right] \cdot\left[\mathrm{VR}_{2}\right]$ as

$$
\begin{array}{r}
\mathbf{X}_{E} \equiv\left(\left[\mathrm{R}_{1}\right],\left[\mathrm{R}_{2}\right],\left[\mathrm{RR}_{1}\right],\left[\mathrm{RR}_{2}\right]\left[\mathrm{VR}_{1}\right],\left[\mathrm{VR}_{2}\right],\left[\mathrm{VRR}_{1}\right],\left[\mathrm{VRR}_{2}\right],\left[\mathrm{RVR}_{1}\right],\left[\mathrm{RVR}_{2}\right]\right. \\
\left.\left[\Delta_{1}\right],\left[\Delta_{2}\right],\left[\mathrm{R}_{1}\right]^{2},\left[\mathrm{R}_{2}\right]^{2},\left[\mathrm{R}_{1}\right] \cdot\left[\mathrm{VR}_{1}\right],\left[\mathrm{R}_{2}\right] \cdot\left[\mathrm{VR}_{2}\right]\right)^{T}
\end{array}
$$

As the rate law vector $\Phi(\mathbf{X})=\left(\phi_{11}, \phi_{12}, \phi_{21}, \cdots, \phi_{71}, \phi_{72}, \phi_{1}, \cdots, \phi_{6}\right)^{T}$ is a linear combination of $\mathbf{X}_{E}$, it can be interpreted as a linear expression: $\Phi(\mathbf{X})=\mathbf{A}_{E} \cdot \mathbf{X}_{E}$. So we have

$$
\frac{d \mathbf{X}}{d t}=\Gamma \cdot \Phi(\mathbf{X})=\Gamma \cdot \mathbf{A}_{E} \cdot \mathbf{X}_{E}=\overline{\mathbf{A}}_{E} \cdot \mathbf{X}_{E},
$$

where $\overline{\mathbf{A}}_{E}=\Gamma \cdot \mathbf{A}_{E}$ (the $12 \times 16$ dimensional expanded system matrix $\overline{\mathbf{A}}_{E}$ is too large to reproduce within normal text). We substitute $X_{1} \equiv\left[\mathrm{R}_{1}\right]^{2}, X_{2} \equiv\left[\mathrm{R}_{2}\right]^{2}, Y_{1} \equiv\left[\mathrm{R}_{1}\right] \cdot\left[\mathrm{VR}_{1}\right]$ and $Y_{2} \equiv\left[\mathrm{R}_{2}\right] \cdot\left[\mathrm{VR}_{2}\right]$ into $\mathbf{X}_{E}$, and denote the new vector as $\overline{\mathbf{X}}_{E}$. Then all elements in $\overline{\mathbf{X}}_{E}$ are linear variables, and the steady state problem is equivalent to that for a linear system $\frac{d \mathbf{X}}{d t}=\Gamma \cdot \Phi(\mathbf{X})=\overline{\mathbf{A}}_{E} \cdot \overline{\mathbf{X}}_{E}$, find the set of $\overline{\mathbf{X}}_{E}$ such that

$$
\frac{d \mathbf{X}}{d t}=0 \Leftrightarrow \overline{\mathbf{A}}_{E} \cdot \overline{\mathbf{X}}_{E}=0 .
$$

By Theorem 1 in [18], $\overline{\mathbf{A}}_{E}$ has the same rank as the original system, i.e. $\operatorname{rank}\left(\overline{\mathbf{A}}_{E}\right)=$ $\operatorname{rank}(\Gamma)=11$. For the linear equation system $\overline{\mathbf{A}}_{E} \cdot \overline{\mathbf{X}}_{E}=0$, as $\operatorname{rank}\left(\overline{\mathbf{A}}_{E}\right)=11$, and there are 16 variables in $\overline{\mathbf{X}}_{E}$, we can solve 11 variables (dependent variables) as a function of the other 5 
(free variables). To achieve that, we first discard a row of $\overline{\mathbf{A}}_{E}$, whose loss would not reduce the rank of $\overline{\mathbf{A}}_{E}$. In this case, we select row 1 .

Next, we select the 11 dependent variables. The set of dependent variables has to be determined carefully to make the method given in [18] work. We select $\overline{\mathbf{X}}_{D}=\left(\left[\mathrm{RR}_{1}\right],\left[\mathrm{RR}_{2}\right],\left[\mathrm{VR}_{1}\right]\right.$, $\left.\left[\mathrm{VR}_{2}\right],\left[\mathrm{VRR}_{1}\right],\left[\mathrm{VRR}_{2}\right],\left[\mathrm{RVR}_{1}\right],\left[\mathrm{RVR}_{2}\right],\left[\Delta_{1}\right],\left[\Delta_{2}\right], Y_{2}\right)^{T}$ as the set of dependent variables, and $\overline{\mathbf{X}}_{F}=\left(\left[\mathrm{R}_{1}\right],\left[\mathrm{R}_{2}\right], X_{1}, X_{2}, Y_{1}\right)^{T}$ as the set of free variables. We use Cramer's Rule to solve for $\overline{\mathbf{X}}_{D}$ in terms of $\overline{\mathbf{X}}_{F}$. Denote the solution as

$$
y_{i}=a_{i 1}\left[\mathrm{R}_{1}\right]+a_{i 2}\left[\mathrm{R}_{2}\right]+a_{i 3} X_{1}+a_{i 4} X_{2}+a_{i 5} Y_{1}
$$

where $i=1,2, \cdots, 11, y_{i} \in X_{D}$ and $a_{i j}$ are algebraic combinations of reaction rate constants. Substitute the bilinears $X_{1}=\left[\mathrm{R}_{1}\right]^{2}, X_{2}=\left[\mathrm{R}_{2}\right]^{2}, Y_{1}=\left[\mathrm{R}_{1}\right]\left[\mathrm{VR}_{1}\right]$ and $Y_{2}=\left[\mathrm{R}_{2}\right]\left[\mathrm{VR}_{2}\right]$ back to $\overline{\mathbf{X}}_{D}$ and $\overline{\mathbf{X}}_{F}$, then the solution can be rewritten as

$$
y_{i}=a_{i 1}\left[\mathrm{R}_{1}\right]+a_{i 2}\left[\mathrm{R}_{2}\right]+a_{i 3}\left[\mathrm{R}_{1}\right]^{2}+a_{i 4}\left[\mathrm{R}_{2}\right]^{2}+a_{i 5}\left[\mathrm{R}_{1}\right]\left[\mathrm{VR}_{1}\right]
$$

where $i=1,2, \cdots, 11$. We carefully select two solutions

$$
\begin{aligned}
& {\left[\mathrm{VR}_{1}\right]=a_{31}\left[\mathrm{R}_{1}\right]+a_{32}\left[\mathrm{R}_{2}\right]+a_{33}\left[\mathrm{R}_{1}\right]^{2}+a_{34}\left[\mathrm{R}_{2}\right]^{2}+a_{35}\left[\mathrm{R}_{1}\right]\left[\mathrm{VR}_{1}\right]} \\
& {\left[\mathrm{VR}_{2}\right]=a_{41}\left[\mathrm{R}_{1}\right]+a_{42}\left[\mathrm{R}_{2}\right]+a_{43}\left[\mathrm{R}_{1}\right]^{2}+a_{44}\left[\mathrm{R}_{2}\right]^{2}+a_{45}\left[\mathrm{R}_{1}\right]\left[\mathrm{VR}_{1}\right] .}
\end{aligned}
$$

It is easy to solve $(5.12)$ for $\left[\mathrm{VR}_{1}\right]$. Denote the solution as $\left[\mathrm{VR}_{1}\right]=\varphi_{1}\left(\left[\mathrm{R}_{1}\right],\left[\mathrm{R}_{2}\right]\right)$. We then substitute the solution of $\left[\mathrm{VR}_{1}\right]$ to $(5.13)$ and let $\left[\mathrm{VR}_{2}\right]=\varphi_{2}\left(\left[\mathrm{R}_{1}\right],\left[\mathrm{R}_{2}\right]\right)$. As

$$
y_{2}=\left[\mathrm{R}_{2}\right]\left[\mathrm{VR}_{2}\right]=a_{11,1}\left[\mathrm{R}_{1}\right]+a_{11,2}\left[\mathrm{R}_{2}\right]+a_{11,3}\left[\mathrm{R}_{1}\right]^{2}+a_{11,4}\left[\mathrm{R}_{2}\right]^{2}+a_{11,5}\left[\mathrm{R}_{1}\right]\left[\mathrm{VR}_{1}\right]
$$

we substitute $\left[\mathrm{VR}_{1}\right]=\varphi_{1}\left(\left[\mathrm{R}_{1}\right],\left[\mathrm{R}_{2}\right]\right)$ and $\left[\mathrm{VR}_{2}\right]=\varphi_{2}\left(\left[\mathrm{R}_{1}\right],\left[\mathrm{R}_{2}\right]\right)$ into $(5.14)$, and this will reduce the variables of (5.14) to $\left[\mathrm{R}_{1}\right]$ and $\left[\mathrm{R}_{2}\right]$. The resulting identity is a cubic function that only has $\left[\mathrm{R}_{1}\right]$ and $\left[\mathrm{R}_{2}\right]$ as variables. By Cardano's method, we solve the cubic function symbolically for $\left[\mathrm{R}_{2}\right]$, and denote the only positive real root by $\left[\mathrm{R}_{2}\right]=\psi\left(\left[\mathrm{R}_{1}\right]\right)$. Substituting $\left[\mathrm{R}_{2}\right]=\psi\left(\left[\mathrm{R}_{1}\right]\right)$ to $\left[\mathrm{VR}_{1}\right]$ solution, we then have

$$
\left[\mathrm{VR}_{1}\right]=\varphi_{1}\left(\left[\mathrm{R}_{1}\right],\left[\mathrm{R}_{2}\right]\right)=\varphi_{1}\left(\left[\mathrm{R}_{1}\right], \psi\left(\left[\mathrm{R}_{1}\right]\right)\right)
$$

As $\left[R_{2}\right]$ and $\left[\mathrm{VR}_{1}\right]$ are expressed as algebraic functions with variable $\left[\mathrm{R}_{1}\right]$, all the variables in $X_{F}=\left\{\left[\mathrm{R}_{1}\right],\left[\mathrm{R}_{2}\right],\left[\mathrm{R}_{1}\right]^{2},\left[\mathrm{R}_{2}\right]^{2},\left[\mathrm{R}_{1}\right]\left[\mathrm{VR}_{1}\right]\right\}$ can be represented as an explicit function of $\left[\mathrm{R}_{1}\right]$, and consequently all the solutions of $(5.11)$ can be rewritten as functions of $\left[\mathrm{R}_{1}\right]$. We expressed all 12 variables as functions of $\left[\mathrm{R}_{1}\right]$. With the conservation law

$$
\begin{aligned}
& R_{\text {total }}=\left[\mathrm{R}_{1}\right]+\left[\mathrm{R}_{2}\right]+2\left[\mathrm{RR}_{1}\right]+2\left[\mathrm{RR}_{2}\right]+\left[\mathrm{VR}_{1}\right]+\left[\mathrm{VR}_{2}\right]+2\left[\mathrm{VRR}_{1}\right]+2\left[\mathrm{VRR}_{2}\right] \\
&+2\left[\mathrm{RVR}_{1}\right]+2\left[\mathrm{RVR}_{2}\right]+2\left[\Delta_{1}\right]+2\left[\Delta_{2}\right],
\end{aligned}
$$


we can solve the equation for $\left[R_{1}\right]$ numerically for any given value of $R_{\text {total }}$. For all parameter values used in this paper, the dependence on $[R]$ was consistent with a single real root, leading us to the conclusion that the system had a unique steady state. This does not exclude the possibility of multiple steady states for other parameter sets.

\subsection{Results and discussion}

We obtained steady states for the differential equations (5.8) by numerically solving the steady state equations as outlined above, for various values of the relative size of the high density area $(f=5 \% \ldots 30 \%)$, the attractiveness parameter $(\alpha=1 \ldots 10)$, and for VEGF concentrations ranging from $0.01 \mathrm{nM}$ to $5 \mathrm{nM}$. We considered three situations for the relative mobility of dimers $\beta=0.5,0.25,0$ (note that the $\beta=0.5$ case corresponds to equivalent monomer and dimer mobilities).

We first performed calculations using the full model of [16]. The equilibrium values for the total number of receptors and signaling complexes in the two domains, as a function of the three parameters $\left(\alpha, f, V_{0}\right)$, are shown in Figures 5.5 and 5.6. Not surprisingly, increasing the attractiveness parameter $\alpha$, relative size $f$ of the HD area results in an increasing fraction of receptors and signaling complexes in the high density area. Increasing the concentration of VEGF leads to overall increased singaling but no significant shifts between the domains.

In Figure 5.5, the total amount of signaling complexes increases only weakly as a function of the attractiveness parameter $\alpha$. This set calculations was performed including both the ligandinduced dimerization as well as the pre-dimerization (DPD) mechanism of the Mac GabhannPopel model. The DPD rate constant $b=0.1$ results in a high degree of dimerization (more than $90 \%$ dimers), even in the absence of ligand or a high affinity domain.

We were especially interested in the effect of dimerization on the preferential accumulation of receptors. While the results in Fig. 5.5 and 5.6 indicate that the accumulation effect is stronger when dimers are not allowed to cross domain boundaries, the ligand dose response curves (rightmost panels) show only a marginal effect due to the presence of ligand.

The explanation for the weakness of these effects is the presence of ligand-independent dimerization (or pre-dimerization) in the Mac Gabhann-Popel model, as well as the high value of the on-surface VEGF-receptor binding constant $a_{S}$. The effect of dimerization on clustering is revealed when pre-dimerization is turned off (by setting $b=0.0001$ in the rate laws) and the on-surface ligand binding rate $a_{s}$ is reduced. These results are shown in Fig. 5.7 and 5.8. The signal is clearly increased as the affinity of the HD domain increases. There is an optimum in the size of the HD area (middle panel, Fig. 5.7). The effect on the signal persists when dimers are allowed to move. While the dependence of localization on attractiveness and domain size are similar to the previous set, the depedendence on $V_{0}$ is dramatically different in Fig. 5.8, showing 
Signaling complexes, DPD included
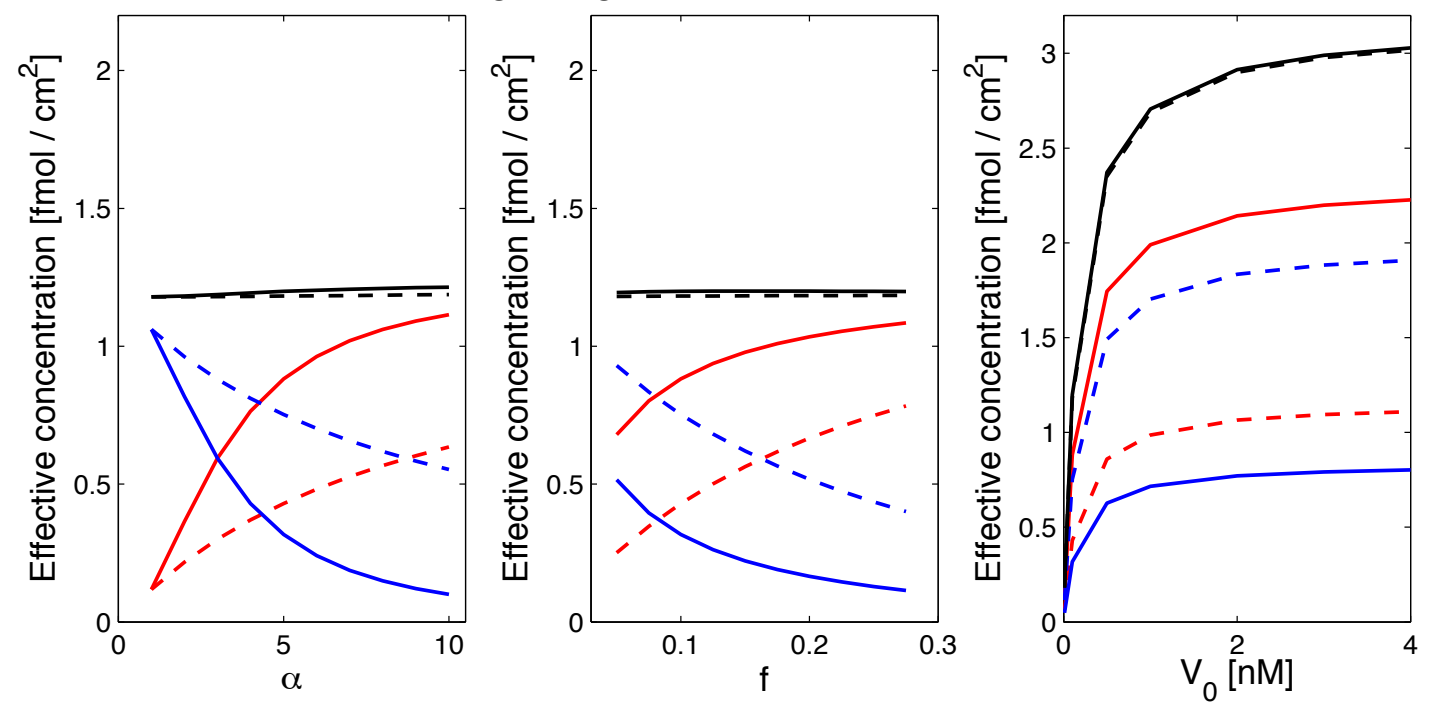

Figure 5.5: Signaling complexes $(R V R$ and $\Delta)$ in the high-affinity (red) and normal (blue) domains, as well as total signal (black) as a function of the attractiveness factor $\alpha$, the relative size of the HD domain $f$, and the concentration of VEGF ligand $V_{0}$. The values for the fixed parameters were $\alpha=5, f=0.1, V_{0}=0.1 \mathrm{nM}$. Solid lines correspond to the case when dimers are not allowed to cross domain boundaries, and dashed lines correspond to fully mobile dimers. The total signal (black lines) depends weakly on the affinity and size of the attractive domain due to the combined effects of the relatively high $\left(V_{0}=0.1 \mathrm{nM}\right)$ VEGF concentration value used in the calculations, as well as due to the presence of strong ligand-independent dimerization (DPD) in the model.

\section{Localization of receptors, DPD included}
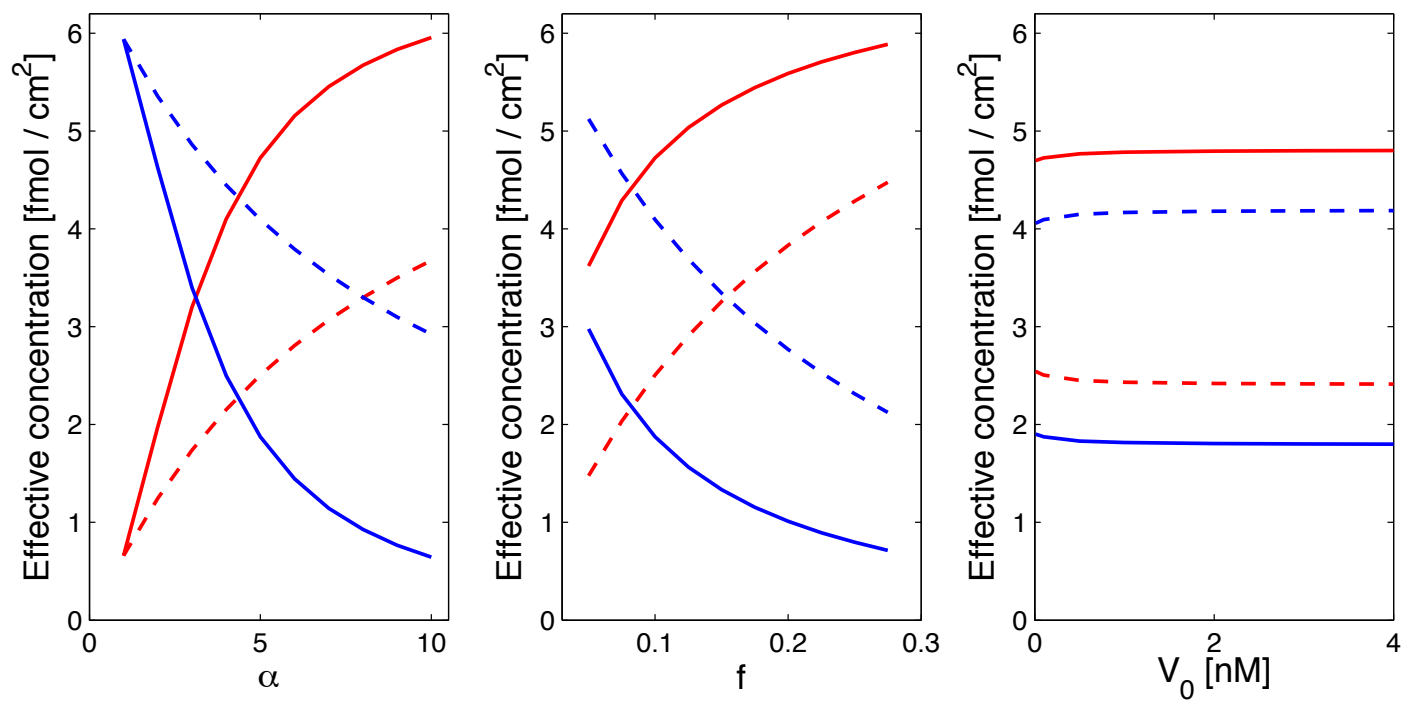

Figure 5.6: Total number of receptors in the high-affinity (red) and normal (blue) domains, in the same set of calculations as in Figure 5.5. The affinity and size of the HD domain strongly influence clustering (represented by the accumulation of receptors in the HD domain), however, the effect of VEGF is marginal, because the model includes ligand-independent dimerization (DPD). 


\section{Signaling complexes, modified model}
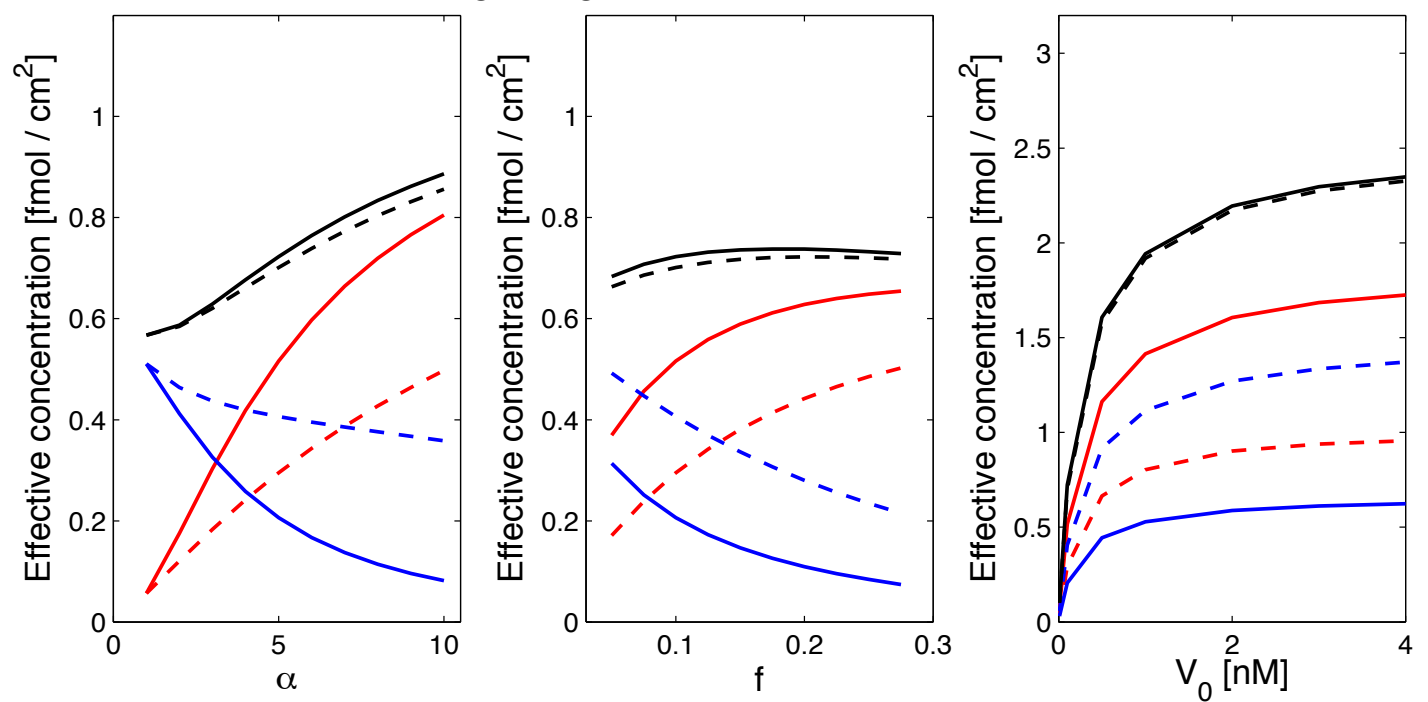

Figure 5.7: Signaling complexes $(R V R$ and $\Delta)$ in the high-affinity (red) and normal (blue) domains, as well as total signal (black) as a function of the attractiveness factor $\alpha$, the relative size of the HD domain $f$, and the concentration of VEGF ligand $V_{0}$. The values for the fixed parameters were $\alpha=5, f=0.1, V_{0}=0.1 \mathrm{nM}$. Solid lines correspond to the case when dimers are not allowed to cross domain boundaries, and dashed lines correspond to fully mobile dimers. This calculation used significantly reduced on-surface dimeriztion rates, namely $a_{s}=0.0021$ and $b=0.0001$ (essentially eliminating DPD). By contrast with Figure 5.5, the affinity of the HD domain strongly enhances the signal, as dimers are formed at a higher rate in the HD domain.

\section{Localization of receptors, modified model}
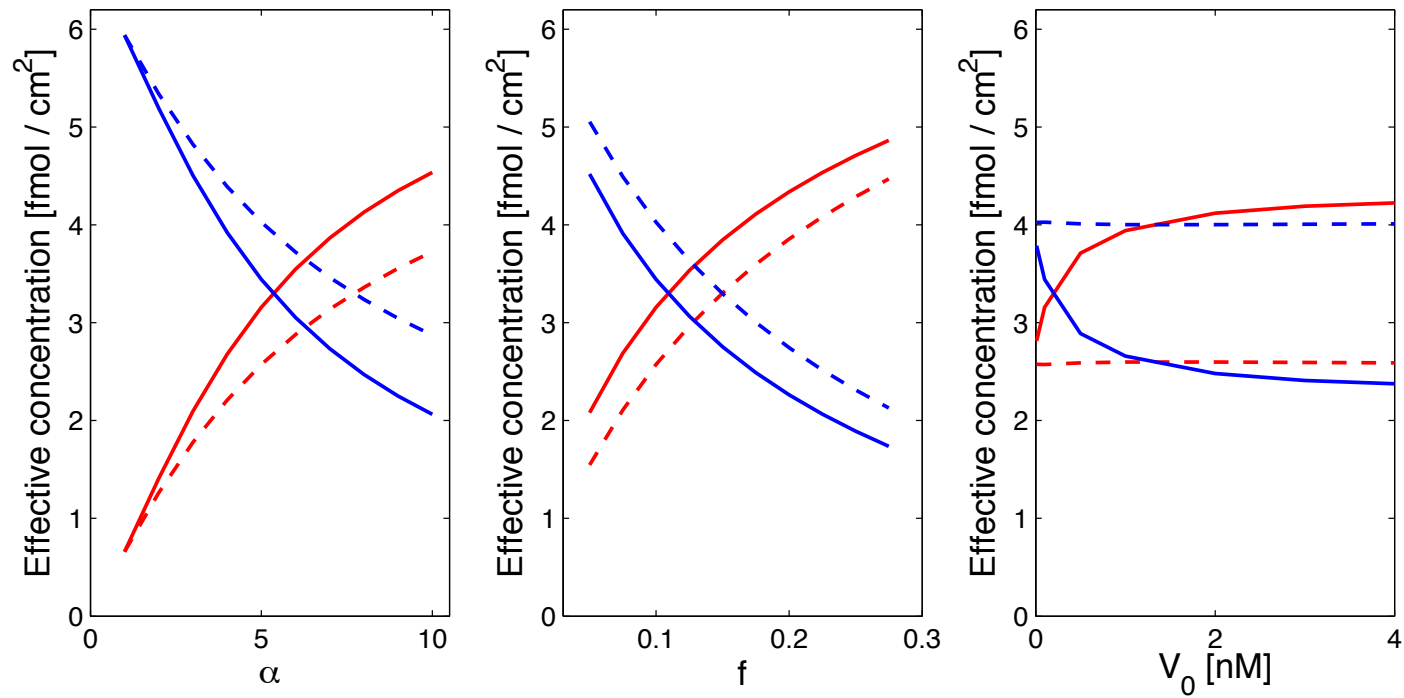

Figure 5.8: Total number of receptors in the high-affinity (red) and normal (blue) domains, in the same set of calculations as in Figure 5.7. By contrast with Figure 5.6, the presence of VEGF strongly promotes the accumulation of receptors in the HD domain. Dimers are formed at a higher rate in the HD domain, and become trapped, causing the HD domain to act as a receptor sink. 
a significant increase in the number of receptors in the HD area as $V_{0}$ is increased. This effect is completely absent when dimers are allowed to move at the same rate as monomers and is very significantly weakened at the intermediate mobility value we used (those results not shown).

In summary, our simple model shows a positive feedback between dimerization / signaling and receptor clustering. Accumulation of receptors in a high affinity patch enhances dimerization and signaling. On the other hand, increased dimerization, in the presence of ligand, inceased the accumulatin of receptors in the high affinity patch. The latter effect also requires a dramatic reduction in the mobility of dimers. Finally, we should point out that the empirically obtained model parameters lead to dimerization rates that are so high that the effects we described here would be marginal; however, the empirically determined parameters are what one would observe as a result of affinity-induced clustering. In other words, in the presence of high density domains, significanly lower dimerization rate constants may be sufficient to achieve the observed signaling. 


\section{Bibliography}

[1] S. Akbari, M. Ghanbari \& S. Jahanbekam (2009): On the list dynamic coloring of graphs. Discrete Appl. Math. 157, pp. 3005-3007.

[2] M. Alishahi (2011): On the dynamic coloring of graphs. Discrete Appl. Math. 159, pp. $152-156$.

[3] N. L. Andrews, K. A. Lidke, J. R. Pfeiffer, A. R. Burns \& B. S. Wilson (2008): Actin restricts FCeRI diffusion and facilitates antigen-induced receptor immobilization. Nat Cell Biol 10, pp. 955-963, doi:10.1038/ncb1755.

[4] K. Appel \& W. Haken (1977): Every plane map is four colorable, Part 1, Reducibility. Illinois J. Math. 21, pp. 429-490.

[5] K. Appel, W. Haken \& J. Kock (1977): Every plane map is four colorable, Part 2, Reducibility. Illinois J. Math. 21, pp. 491-567.

[6] D. A. Birk, J. Barbato, L. Mureebe \& R. A. Chaer (2010): Current insights on the biology and clinical aspects of VEGF regulation. Vasc Endovascular Surg 42, pp. 517-530, doi:10.1177/1538574408322755.

[7] J. A. Bondy \& U. S. R. Murty (2008): Graph Theory.

[8] Y. Chen, S.-H. Fan, H.-J. Lai, H.-M. Song \& L. Sun (2012): On dynamic coloring for planar graphs and graphs of higher genus. Discrete Appl. Math. 160, pp. 1064-1071.

[9] Ye Chen, Christopher Short, Adam M. Halasz \& Jeremy S. Edwards (2013): The impact of high density receptor clusters on VEGF signaling. Electronic Proceedings in Theoretical Computer Science (125), pp. 37-52, doi:10.4204/EPTCS.125.3.

[10] C. Ding, S. Fan \& H.-J. Lai (2008): Upper bound on conditional chromatic number of graphs. J. of Jinan University 29, pp. 7-14.

[11] R. J. Duffin (1965): Topology of series-parallel networks. J. Math. Anal. Appl. 10, pp. 303-318. 
[12] L. Esperet (2010): Dynamic list coloring of bipartite graphs. Discrete Appl. Math. 158, pp. 1963-1965.

[13] Flor A Espinoza, Janet M Oliver \& Bridget S Wilson (2011): Using Hierarchical Clustering and Dendrograms to Quantify the Clustering of Membrane Proteins. Bull. Math. Biol. 74(1), pp. 190-211.

[14] N. Ferrara, K. J. Hilla, H. P. Gerber \& W. Novotny (2004): Discovery and development of bevazicumab, an anti-VEGF antibody for treating cancer. Net Rev Drug Discov 3, pp. 391-400, doi:10.1038/nrd1381.

[15] P. Franklin (1934): A six colour problem. J. Math. Phys. 13, pp. 363-369.

[16] F. Mac Gabhann \& A. S. Popel (2007): Dimerization of VEGF receptors and implications for signal transduction: A computational study. Biophysical Chemistry 128, pp. 125-139, doi:10.1016/j.bpc.2007.03.010.

[17] A. M. Gallegos, S. M. Storey, A. B. Kier, F. Shroeder \& J. M. Ball (2006): Structure and cholesterol dynamics of caveloae/raft and nonraft plasma membrane domains. Biochemistry 45, pp. 12100-12116, doi:10.1021/bi0602720.

[18] Á. M. Halász, H.-J. Lai, M. M. McCabe Pryor, K. Radhakrishnan \& J. S. Edwards (2013): Analytical Solution of Steady State Equations for Chemical Reaction Networks with Bilinear Rate Laws. IEEE/ACM Transactions on Computational Biology and Bioinformatics, doi:10.1109/TCBB.2013.41. Epub before print.

[19] D. Hanahan \& J. Folkman (1996): Patterns and emerging mechanisms of the angiogenetic switch during tumorigenesis. Cell 86, pp. 353-364, doi:10.1016/S0092-8674(00)80108-7.

[20] P. J. Heawood (1890): Map colour theorem. Quart. J. Pure Math. 24, pp. 332-338.

[21] A K Jain, M N Murty \& P J Flynn (1999): ACM Computing Surveys 31(3), pp. 264-323.

[22] W.-F. Wang K.-W. Lih \& X. Zhu (2003): Coloring the square of a $K_{4}$-minor free graph. Discrete Math. 269, pp. 303-309.

[23] A. F. Karamysheva (2008): Mechanisms of angiogenesis. Biochemistry (Mosc) 73, pp. 751-762, doi:10.1134/S0006297908070031.

[24] L. B. Kleiman, T. Maiwald, H. Conzelman, D. A. Lauffenburger \& P. K. Sorger (2011): Rapid phospho-turnover by receptor tyrosine kinases impacts downstream signaling and drug binding. Molecular Cell 43, pp. 723-737, doi:10.1016/j.molcel.2011.07.014. 
[25] H.-J. Lai, J. Lin, B. Montgomery, Zhisui Tao \& S. Fan (2006): Conditional colorings of graphs. Dircrete Math. 306, pp. 1997-2004.

[26] H.-J. Lai, B. Montgomery \& H. Poon (2003): Upper bounds of dynamic chromatic number. Ars Combin. 68, pp. 193-201.

[27] B. F. Lillemeier, J. R. Pfeiffer, Z. Surviladze, B. S. Wilson \& M. M. Davis (2006): Plasma membrane-associated proteins are clustered into islands attached to the cytoskeleton. Proc Natl Acad Sci USA 103, pp. 18992-18997, doi:10.1073/pnas.0609009103.

[28] Y. Lin (2008): Upper bounds of conditional chromatics number. Master thesis, Jinan University.

[29] M. Lohela, M. Bry, T. Tammela \& K. Alitalo (2009): VEGFs and receptors involved in angiogenesis versus lymphangiogenesis. Curr Opin Cell Biol 21, pp. 154-165, doi:10.1016/j.ceb.2008.12.012.

[30] B. Montgomery (2001): Upper bounds of dynamic chromatic number. PhD Dissertation, West Virginia University.

[31] K. Murase, T. Fujiwara, Y. Umemura, K. Suzuki, R. Iino \& H. Murakoshi (2004): Ultrafine membrane compartments for molecular diffusion as revealed by single molecule techniques. Biophys J 75, pp. 4075-4093, doi:10.1529/biophysj.103.035717.

[32] D. A. Olsson, J. Kreuger \& L. Claesson-Welsh (2006): VEGF receptor signaling - in control of vascular function. Nat Rev Mol Cell Biol 7, pp. 359-371, doi:10.1038/nrm1911.

[33] O. Ore (1967): The Four Color Problem. Academic Press, New York.

[34] G. Orr, D. Hu, S. Ozcelik, L. K. Opresko \& H. S. Wiley (2005): Cholesterol dictates the freedom of EGF receptors and HER2 in the plane of the membrane. Biophys $J$ 89, pp. 1362-1373, doi:10.1529/biophysj.104.056192.

[35] T. Parsons, G. Pica, T. Pisanski \& A. Ventre (1987): Orientably simple graphs. Math. Slovaca 37, pp. 391-394.

[36] J. Plouet, J. Schilling \& D. Gospodarowicz (1989): Isolation and characterization of a newly identified endothelial cell mitogen produced by AtT-20 cels. EMBO J 8, pp. 3801-3806.

[37] G. Ringel (1959): Farbungsprobleme auf Frachen und Graphen, Veb Deutscher Verlag Der Wissenschaften.

[38] K. Ritchie \& A. Kusumi (2003): Single-particle tracking image microscopy. Methods Enzymol 360, pp. 618-634, doi:10.1016/S0076-6879(03)60131-X. 
[39] N. Robertson, D. P. Sanders, P. Seymour \& R. Thomas (1997): The Four-Colour Theorem. J. Combin. Theory Ser. B 70(1), pp. 2-44.

[40] R. J. Roskoski (2008): VEGF receptor protein-tyrosine kinases: structure and regulation. Biochem Biophys Res Commun 375, pp. 287-291, doi:10.1016/j.bbrc.2008.07.121.

[41] F. Schoreder, A. M. Gallegos, B. P. Atshaves, S. M. Storey \& A. L. McIntosh (2001): Recent advances in membrane microdomains: rafts, caveolae, and intracellular cholesterol trafficking. Exp Biol Med (Maywood) 226, pp. 873-890.

[42] D. R. Senger, S. J. Galli, A. M. Dvorak, C. A. Perruzzi, V. S. Harvey \& H. F. Dvorak (1983): Tumor cells secrete a vascular permeability factor that promotes accumulation of ascites fluid. Science 219, pp. 983-985, doi:10.1126/science.6823562.

[43] S. J. Singer \& G. L. Nicholson (1972): The Fluid Mosaic Model of the Structure of Cell Membranes. Science 175, pp. 720-731, doi:10.1126/science.175.4023.720.

[44] E. Stuttfield \& K. Ballmer-Hofer (2009): Structure and Function of VEGF Receptors. IUBMB Life 61(9), pp. 915-922, doi:10.1002/iub.234.

[45] G. Vereb, J. Szollosi, J. Matko, P. Nagy, T. Farkas, L. Vigh, L. Matyus, T. A. Waldmann \& S. Djanovich (2003): Dynamic, yet structured: The cell membrane three decades after the Singer-Nicolson model. Proc Natl Acad Sci USA 100(14), pp. 8053-8058, doi:10.1073/pnas. 1332550100.

[46] G. Wegner (1977): Graphs with given diameter and a coloring problem. Technical Report, University of Dortmund.

[47] A.T. White (1973): Graphs, Groups and Surfaces.

[48] B. S. Wilson, J. R. Pfeiffer, M. A. Raymond-Stintz, D. Lidke \& N. Andrews (2007): Exploring membrane domains using native membrane sheets and transmission electron microscopy. Methods Mol Biol 398, pp. 245-261, doi:10.1007/978-1-59745-513-8_17.

[49] J. W. T. Youngs (1963): Minimal imbedding and the genus of a graph. J. Math. Mech. 12, pp. 303-315.

[50] Jun Zhang, Karin Leiderman, Janet R. Pfeiffer, Bridget S. Wilson, Janet M. Oliver \& Stanly L. Steinberg (2006): Characterizing the topography of membrane receptors and signaling molecules from spatial patterns obtained using nanometer-scale electron-dense probes and electron microscopy. Micron 37, pp. 14-34, doi:10.4204/EPTCS.125.3. 